\title{
Indicators of Similarity and Dissimilarity of Multi-Attribute Objects in the Metric Spaces of Sets and Multisets
}

\author{
A. B. Petrovsky ${ }^{a, b, c, *}$ \\ ${ }^{a}$ Federal Research Center "Computer Science and Control”, Russian Academy of Sciences, Moscow, 119333 Russia \\ ${ }^{b}$ National Research University Belgorod State University, Belgorod, 308015 Russia \\ ${ }^{c}$ Shukhov Belgorod State Technological University, Belgorod, 308012 Russia \\ *e-mail:pab@isa.ru
}

\begin{abstract}
The concepts of the similarity and dissimilarity (difference) of analyzed objects play an important role in many theoretical and practical problems of decision making, artificial intelligence, pattern recognition, processing of heterogeneous information, etc. The similarity or dissimilarity of objects is usually estimated by their proximity in the attribute space. This paper considers new classes of metric spaces of finite, bounded, and measurable sets and multisets. The possibilities are shown for using new types of metrics to evaluate the similarity or dissimilarity of multi-attribute objects that are present in several instances with differing values of attributes and are represented by multisets of attributes.
\end{abstract}

Keywords: multi-attribute objects, similarity and dissimilarity of objects, multiset, metric spaces of sets and multisets, metrics, pseudometrics, quasi-metrics

DOI: $10.3103 / \mathrm{S} 0147688218050052$

\section{INTRODUCTION}

In various theoretical and practical problems of decision making, artificial intelligence, pattern recognition, data processing, and in other subject areas there is often a need to estimate the similarity or dissimilarity of studied objects (variants or alternatives) on the basis of their properties, which are expressed by attributes of objects. The similarity or dissimilarity of objects is usually estimated by their proximity in the attribute space.

There is a rather wide range of problems where the analyzed objects are given by many heterogeneous attributes, that is, quantitative, qualitative, mixed, continuous or discrete ones; as well, the same objects are present in several instances (versions or copies) with different values of attributes, whose convolution is either impossible or mathematically incorrect. Different versions of object description arise in cases when an object is estimated by several experts for many criteria with numerical and/or verbal scales, or the characteristics of the object are calculated several times by different methods, or measured several times by different instruments. Examples of such problems are group classification and group ordering of multiattribute decisions, recognition of graphic symbols, and processing of text documents. The multiplicity and repetition of factors that describe objects complicates and hinders the solution of such problems.

A convenient mathematical model for representing the multi-attribute objects is a multiset or a set with repeating elements. The multiplicity of elements is an essential attribute of the multiset, which distinguishes it from the set. This paper considers new types of metric spaces of sets and multisets. The possibilities of application of new types of pseudometrics and quasimetrics for estimating the similarity or dissimilarity of multi-attribute objects that are present in several instances with differing attribute values and are represented by multisets of attributes are shown.

\section{MULTISETS AND OPERATIONS ON MULTISETS}

Let us briefly outline the main theses of the theory of multisets [6,7]. A multiset $\boldsymbol{A}$ generated by an ordinary (crisp) set $X=\left\{x_{1}, x_{2}, \ldots\right\}$, all elements $x_{i}$ of which are distinct, is defined as a set of groups of identical elements written in the form

$$
\boldsymbol{A}=\left\{k_{A}(x) \circ x \mid x \in X, K_{A}(x) \in \mathbb{Z}_{+}\right\} .
$$

Here, $k_{A}: X \rightarrow \mathbb{Z}_{+}=\{0,1,2, \ldots\}$ is called a function of the number of instances of a multiset, which specifies the multiplicity of the occurrence of element $x \in X$ in multiset $\boldsymbol{A}$ that is denoted by symbol $\circ$. The multiset is a generalization of the concept of a set and becomes a set for $k_{A}(x)=\chi_{A}(x)$, where $\chi_{A}(x)=1$ for $x \in A$ and $\chi_{A}(x)=0$ for $x \notin A$. Group $k_{A}(x) \circ x=x^{k_{A}(x)}$ that unites $k$ identical elements $x$ is called a component of multiset $\boldsymbol{A}$. A one-element set $\{x\}=\left\{x^{1}\right\}=\{1 \circ x\}$ is called a simple 
set or a singleton, while a one-component multiset $\left\{x^{k}\right\}$ $=\{k \circ x\}$ is called a simple multiset, multiple singleton, or multiton. A "zero-point" set $\left\{x^{0}\right\}=\{0 \circ x\}$ is called a zeron.

The set $\operatorname{Supp} \boldsymbol{A}=\left\{x \in A \mid \chi_{\operatorname{Supp} \boldsymbol{A}}(x)=\min \left[k_{A}(x), 1\right]\right\}$ is called a carrier or a support set of the multiset $\boldsymbol{A}$. The cardinality of a multiset is defined as the total number of all instances of elements of multisets, equal to the sum of their multiplicities: $\operatorname{card} \boldsymbol{A}=|\boldsymbol{A}|=\sum_{x} k_{A}(x)$; the dimension of a multiset is defined as the total number of single instances of all elements of a multiset, equal to the sum of their single multiplicities: $\operatorname{dim} \boldsymbol{A}=|\boldsymbol{A}|=$ $\sum_{x} \chi_{A}(x)=|\operatorname{Supp} A|$. The maximum value of the multiplicity function alt $\boldsymbol{A}=\max _{x \in X^{\prime}} k_{A}(x)$ is called the height of multiset $\boldsymbol{A}$.

Multisets $\boldsymbol{A}$ and $\boldsymbol{B}$ are said to be equal $(\boldsymbol{A}=\boldsymbol{B})$ when $k_{A}(x)=k_{B}(x), \forall x \in X$. Multisets $\boldsymbol{A}$ and $\boldsymbol{B}$ are unequal $(\boldsymbol{A} \neq \boldsymbol{B})$ when $k_{A}(x) \neq k_{\boldsymbol{B}}(x)$ for at least one $x \in X$. For equal multisets, we have $|\boldsymbol{A}|=|\boldsymbol{B}|,|\boldsymbol{A}|=\mid \boldsymbol{B} /, \operatorname{Supp} \boldsymbol{A}=$ Supp $\boldsymbol{B}$, alt $\boldsymbol{A}=$ alt $\boldsymbol{B}$. Multisets $\boldsymbol{A}$ and $\boldsymbol{B}$ are said to be of equal cardinality if $|\boldsymbol{A}|=|\boldsymbol{B}|$; of equal dimension if $|\boldsymbol{A}|=$ $/ \boldsymbol{B} /$; of equal size if they are of equal cardinality and equal dimension. Equal multisets are equal in size, the converse statement, generally speaking, is not true. The equality of multisets is an equivalence relation, since it is reflexive $(\boldsymbol{A}=\boldsymbol{A})$, symmetric $(\boldsymbol{A}=\boldsymbol{B}, \boldsymbol{B}=\boldsymbol{A})$, and transitive $(\boldsymbol{A}=\boldsymbol{B}, \boldsymbol{B}=\boldsymbol{C} \Rightarrow \boldsymbol{A}=\boldsymbol{C})$.

It is said that multiset $\boldsymbol{A}$ is included in multiset $\boldsymbol{B}$ $(\boldsymbol{A} \subseteq \boldsymbol{B})$ when $k_{\boldsymbol{A}}(x) \leq k_{\boldsymbol{B}}(x), \forall x \in X$. Multiset $\boldsymbol{A}$ is then called a submultiset or a multisubset of multiset $\mathbf{B}$, and multiset $\mathbf{B}$ is an overmultiset or an initial, parent multiset of multiset $\boldsymbol{A}$. In this case $|\boldsymbol{A}| \leq|\boldsymbol{B}|, \mid \boldsymbol{A} / \leq / \boldsymbol{B} /$, Supp $\boldsymbol{A} \subseteq$ Supp $\boldsymbol{B}$, alt $\boldsymbol{A} \subseteq$ alt $\boldsymbol{B}$. As in the case of sets, the simultaneous fulfillment of conditions $\boldsymbol{A} \subseteq \boldsymbol{B}$ and $\boldsymbol{B} \subseteq \boldsymbol{A}$ implies the equality of multisets $\boldsymbol{A}=\boldsymbol{B}$. If $\boldsymbol{A} \subseteq \boldsymbol{B}$, but

$\boldsymbol{B} \nsubseteq \boldsymbol{A}$, then multiset $\boldsymbol{A}$ is called the proper submultiset of multiset $\boldsymbol{B}$ and denoted as $\boldsymbol{A} \subset \boldsymbol{B}$. The inclusion of the multiset is the preorder relation, since it is reflexive $(\boldsymbol{A} \subseteq \boldsymbol{A})$ and transitive $(\boldsymbol{A} \subseteq \boldsymbol{B}, \boldsymbol{B} \subseteq \boldsymbol{C} \Rightarrow \boldsymbol{A} \subseteq \boldsymbol{C})$.

Multiset $\boldsymbol{Z}$ is called maximal if all multisets $\boldsymbol{A}$ of the family of multisets $\boldsymbol{A}$ are submultisets of multiset $\boldsymbol{Z}$ $(A \subseteq Z)$, that is, if $k_{A}(x) \leq k_{Z}(x)$; empty $\varnothing$ if $k_{\varnothing}(x)=0$; constant $N_{[h]}$ if $k_{N_{[h]}}(x)=h=$ const, $h \in \mathrm{Z}_{+}, \forall x \in X$. Thus, the empty multiset $\varnothing$ is a constant multiset $N_{\text {[0] }}$ of height 0 , and the common set $\boldsymbol{A}$, in particular, the support $\operatorname{Supp} \boldsymbol{A}$ of an arbitrary multiset $\boldsymbol{A}$, is a constant multiset $N_{[1]}$ of height 1. The maximal multiset $\boldsymbol{Z}$ can also be considered as a constant multiset $N_{[k]}$ of height $k=k_{Z}(x)=\max _{\boldsymbol{A} \in A} k_{A}(x), \forall x \in X$.

Let us assume that all the multisets that constitute family $\boldsymbol{A}=\left\{\boldsymbol{A}_{i}\right\}_{i \in I}$ are submultisets of the same maximal multiset $\boldsymbol{Z}$ generated by some set $X$. Let us define the following operations on multisets:

union

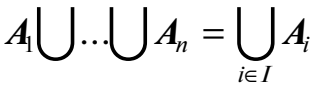

$=\left\{k_{\cup_{i} A_{i}}(x) \circ x \mid k_{\cup_{i} A_{i}}(x)=\max _{i \in I} k_{A_{i}}(x), \forall x \in X\right\}$; intersection

$$
A_{1} \bigcap \ldots \bigcap A_{n}=\bigcap_{i \in I} A_{i}
$$

$=\left\{k_{\bigcap_{i} A_{i}}(x) \circ x \mid k_{\bigcap_{i} A_{i}}(x)=\min _{i \in I} k_{A_{i}}(x), \forall x \in X\right\}$;

addition

$$
\begin{gathered}
\boldsymbol{A}_{1}+\ldots+\boldsymbol{A}_{n}=\sum_{i \in I} \boldsymbol{A}_{i} \\
=\left\{k_{\sum_{i} A_{i}}(x) \circ x \mid k_{\sum_{i} A_{i}}(x)=\sum_{i \in I} k_{A_{i}}(x), \forall x \in X\right\} ;
\end{gathered}
$$

subtraction

$$
\begin{aligned}
& \boldsymbol{A}-\boldsymbol{B}=\left\{k_{A-\boldsymbol{B}}(x) \circ x \mid k_{A-\boldsymbol{B}}(x)\right. \\
& \left.=\max \left[k_{\boldsymbol{A}}(x)-k_{\boldsymbol{B}}(x), 0\right], \forall x \in X\right\} ;
\end{aligned}
$$

symmetric difference

\section{$\boldsymbol{A} \Delta \boldsymbol{B}$}

$=\left\{k_{A \Delta B}(x) \circ x\left|k_{A \Delta B}(x)=\right| k_{A}(x)-k_{B}(x) \mid, \forall x \in X\right\} ;$ complement

$$
\begin{aligned}
\overline{\boldsymbol{A}} & =\boldsymbol{Z}-\boldsymbol{A} \\
=\left\{k_{\bar{A}}(x) \circ x \mid k_{\bar{A}}(x)\right. & \left.=k_{Z}(x)-k_{\boldsymbol{A}}(x), \forall x \in X\right\} ;
\end{aligned}
$$

multiplication by a scalar (reproduction)

\section{$b \cdot \boldsymbol{A}$}

$=\left\{k_{b \cdot A}(x) \circ x \mid k_{b \cdot A}(x)=b \cdot k_{A}(x), b \in \mathbb{Z}_{+}, \forall x \in X\right\} ;$ multiplication

$$
\begin{gathered}
\boldsymbol{A}_{1} \cdot \ldots \cdot \boldsymbol{A}_{n}=\prod_{i \in I} \boldsymbol{A}_{i} \\
=\left\{k_{\Pi_{i} \boldsymbol{A}_{i}}(x) \circ x \mid k_{\prod_{i} \boldsymbol{A}_{i}}(x)=\prod_{i \in I} k_{\boldsymbol{A}_{i}}(x), \forall x \in X\right\} ; \\
n \text {-th power } \\
\boldsymbol{A}^{n}=\left\{k_{\boldsymbol{A}^{n}}(x) \circ x \mid k_{\boldsymbol{A}^{n}}(x)=\left(k_{\boldsymbol{A}}(x)\right)^{n}, \forall x \in X\right\} ;
\end{gathered}
$$

direct product

$$
\begin{gathered}
A_{1} \times \ldots \times A_{n} \\
=\left\{k_{A_{1} \times \ldots \times A_{n}}\left(x_{p_{1}}, \ldots, x_{p_{n}}\right) \circ\left\langle x_{p_{1}}, \ldots, x_{p_{n}}\right\rangle \mid k_{A_{1} \times \ldots \times A_{n}}\left(x_{p_{1}}, \ldots, x_{p_{n}}\right)\right. \\
\left.=\prod_{i} k_{A_{i}}\left(x_{p_{i}}\right), x_{p_{i}} \in A_{i}, i=1, \ldots, n\right\} ;
\end{gathered}
$$


direct $n$-th power

$$
(\times \boldsymbol{A})^{n}=\left\{k_{(\times \boldsymbol{A})^{n}}(x) \circ x \mid k_{(\times A)^{n}}(x)=\prod_{i} k_{\boldsymbol{A}}\left(x_{i}\right), x_{i} \in \boldsymbol{A}, i=1, \ldots n\right\} .
$$

The supports of operations on multisets satisfy the following relations:

$$
\begin{gathered}
\operatorname{Supp}(\boldsymbol{A} \bigcup \boldsymbol{B})=\operatorname{Supp}(\boldsymbol{A}+\boldsymbol{B}) \\
=(\operatorname{Supp} \boldsymbol{A}) \bigcup(\operatorname{Supp} \boldsymbol{B}) ; \\
\operatorname{Supp}(\boldsymbol{A} \bigcap \boldsymbol{B})=\operatorname{Supp}(\boldsymbol{A} \cdot \boldsymbol{B}) \\
=(\operatorname{Supp} \boldsymbol{A}) \bigcap(\operatorname{Supp} \boldsymbol{B}) ; \\
\operatorname{Supp}(\boldsymbol{A \Delta B})=(\operatorname{Supp}(\boldsymbol{A}-\boldsymbol{B})) \bigcup(\operatorname{Supp}(\boldsymbol{B}-\boldsymbol{A})) ; \\
\operatorname{Supp}(b \cdot \boldsymbol{A})=\operatorname{Supp} \boldsymbol{A}=\operatorname{Supp}\left(\boldsymbol{A}^{n}\right) ; \\
\operatorname{Supp}(\boldsymbol{A} \times \boldsymbol{B})=(\operatorname{Supp} \boldsymbol{A}) \times(\operatorname{Supp} \boldsymbol{B}) ; \\
\operatorname{Supp}(\times \boldsymbol{A})^{n}=(\times \operatorname{Supp} \boldsymbol{A})^{n} .
\end{gathered}
$$

Many of the properties of operations on multisets are analogous to the properties of operations on ordinary sets, including the idempotency of union and intersection $\boldsymbol{A} \bigcup \ldots \bigcup \boldsymbol{A}=\boldsymbol{A}, \boldsymbol{A} \bigcap \ldots \bigcap \boldsymbol{A}=\boldsymbol{A}$, involution (double negation) of complementation $\overline{\bar{A}}=\boldsymbol{A}$, identity, commutativity, associativity, and distributivity of some operations. As in the case of sets, not all operations on multisets are mutually commutative, associative, and distributive. In addition, operations of addition, multiplication by a scalar, multiplication, and raising to an arithmetic power are not defined for sets. In the transition from multisets to sets, operations of multiplication and raising to an arithmetic power turn into intersection, and operations of addition and multiplication by a scalar become impossible.

For multisets, as well as for sets, there is a duality of the operations of union and intersection in relation to the complementation operation, similar to the laws of De Morgan, and, in addition, there is a new type of duality of operations of arithmetic addition and subtraction:

$$
\begin{gathered}
\overline{A \bigcup B}=\bar{A} \bigcap \bar{B}, \overline{A \bigcap B}=\bar{A} \bigcup \bar{B} \\
\bar{A}+\boldsymbol{B}=\bar{A}-B=\bar{B}-A \\
\overline{A-B}=\bar{A}+B, \quad \bar{A}-\bar{B}=B-A .
\end{gathered}
$$

Multisets lack some of the properties of operations that sets have. At the same time, new properties appear that have no analogues for sets. As an example, in the theory of sets, the following equalities are always satisfied: $A \bigcap \bar{A}=\varnothing, A \bigcup \bar{A}=U$, where $U$ is a universal set. Unlike sets, for multisets the following relations, which follow from the definition of complementation of a multiset, are satisfied: $\boldsymbol{A}+\overline{\boldsymbol{A}}=\boldsymbol{Z}, \boldsymbol{Z}-\overline{\boldsymbol{A}}=\boldsymbol{A}$. At the same time, both $\boldsymbol{A} \bigcap \overline{\boldsymbol{A}}=\varnothing, \boldsymbol{A} \bigcup \overline{\boldsymbol{A}}=\boldsymbol{Z}, \boldsymbol{A}-\overline{\boldsymbol{A}}=$ $\bar{A}-\boldsymbol{A}=\varnothing$ and $\boldsymbol{A} \bigcap \overline{\boldsymbol{A}} \neq \varnothing, \boldsymbol{A} \bigcup \overline{\boldsymbol{A}} \neq \boldsymbol{Z}, \boldsymbol{A}-\overline{\boldsymbol{A}} \neq \overline{\boldsymbol{A}}-$ $\boldsymbol{A} \neq \varnothing$ (there are also possible cases where $\boldsymbol{A} \subseteq \overline{\boldsymbol{A}}$ and where $\overline{\boldsymbol{A}} \subseteq \boldsymbol{A})$. In addition, the empty multiset $\varnothing$ and the maximal multiset $\boldsymbol{Z}$ are mutually complementary: $\varnothing=\bar{Z}, \boldsymbol{Z}=\bar{\varnothing}$, and so $\varnothing \bigcap \bar{\varnothing}=\boldsymbol{Z} \bigcap \overline{\boldsymbol{Z}}=\varnothing$.

Another very specific attribute of operations on multisets of family $\boldsymbol{A}=\left\{\boldsymbol{A}_{i}\right\}_{i \in I}$ is the possibility of their linear combinations with the operation of multiplication by a scalar (reproduction), for example:

weighted union

$$
\begin{aligned}
& \bigcup_{i \in I}\left(b_{i} \cdot A_{i}\right)=\left\{k_{\cup_{i}\left(b_{i} \cdot A_{i}\right)}(x) \circ x \mid k_{\cup_{i}\left(b_{i} \cdot A_{i}\right)}(x)\right. \\
& \left.=\max _{i \in I}\left(b_{i} \bullet k_{A_{i}}(x)\right), b_{i} \geq 1, \forall x \in X\right\} ;
\end{aligned}
$$

weighted intersection

$$
\begin{gathered}
\bigcap_{i \in I}\left(b_{i} \cdot A_{i}\right)=\left\{k_{\cap_{i}\left(b_{i} \cdot A_{i}\right)}(x) \circ x \mid k_{\bigcap_{i}\left(b_{i} \cdot A_{i}\right)}(x)\right. \\
\left.=\min _{i \in I}\left(b_{i} \cdot k_{A_{i}}(x)\right), b_{i} \geq 1, \forall x \in X\right\} ;
\end{gathered}
$$

weighted arithmetic sum

$$
\begin{gathered}
\sum_{i \in I}\left(b_{i} \bullet A_{i}\right)=\left\{k_{\sum_{i}\left(b_{i} \cdot A_{i}\right)}(x) \circ x \mid k_{\sum_{i}\left(b_{i} \cdot A_{i}\right)}(x)\right. \\
\left.=\sum_{i \in I}\left(b_{i} \bullet k_{A_{i}}(x)\right), b_{i} \geq 1, \forall x \in X\right\} ;
\end{gathered}
$$

weighted arithmetic product

$$
\begin{gathered}
\prod_{i \in I}\left(b_{i} \cdot A_{i}\right)=\left\{k_{\prod_{i}\left(b_{i} \cdot A_{i}\right)}(x) \circ x \mid k_{\Pi_{i}\left(b_{i} \cdot A_{i}\right)}(x)\right. \\
\left.=\prod_{i \in I}\left(b_{i} \cdot k_{A_{i}}(x)\right), b_{i} \geq 1, \forall x \in X\right\} ;
\end{gathered}
$$

weighted direct product

$$
\begin{gathered}
\left(b_{1} \cdot A_{1}\right) \times \ldots \times\left(b_{n} \cdot A_{n}\right) \\
=\left\{k_{\left(b_{1} \cdot A_{1}\right) \times \ldots \times\left(b_{n} \cdot A_{n}\right)}\left(x_{p_{1}}, \ldots, x_{p_{n}}\right) \circ\left\langle x_{p_{1}}, \ldots, x_{p_{n}}\right\rangle,\right. \\
\left.b_{i} \geq 1, \forall x \in X\right\} ;
\end{gathered}
$$

where the function of the number of instances is given by

$$
\begin{gathered}
k_{\left(b_{1} \cdot A_{1}\right) \times \ldots \times\left(b_{n} \cdot A_{n}\right)}\left(x_{p_{1}}, \ldots, x_{p_{n}}\right)=\prod_{i}\left(b_{i} \bullet k_{A_{i}}\left(x_{p_{i}}\right)\right), \\
x_{p_{i}} \in A_{i}, i=1, \ldots, n .
\end{gathered}
$$

When $b_{i}=1$, the "weighted" operations turn into the corresponding "unweighted" operations on multi- 
sets. The possibility of introducing and using linear combinations of operations significantly increases the array of means of operating with multisets. When turning from multisets to sets and replacing the multiplicity function $k_{A}(x)$ by the characteristic function $\chi_{A}(x)$, many of the above statements for multisets remain valid for sets, but some can become indeterminate, change, or lose meaning.

\section{FAMILIES AND FUNCTIONS OF MULTISETS}

Let us consider some special types of families and functions of multisets $[6,7,11]$.

The family of all subsets of set $A$ (power set), which includes set $A$ itself and the empty set $\varnothing$, is called a Boolean of set $A$ and is denoted by $P(A)$. Boolean $P(A)$ is a set of all subsets of set $A$.

A family of all various submultisets of multiset $\boldsymbol{A}$ over domain $X$ (macroset) is called a macroset or a Boolean of multiset $\boldsymbol{A}$ and is denoted by $\boldsymbol{P}(\boldsymbol{A})$. The word various plays an important role here, since the theory of multisets allows, generally speaking, unlimited repeatability of elements in the same multiset and, accordingly, the repeatability of multisets in the same family of multisets. In family $\boldsymbol{P}(\boldsymbol{A})$, various subsets are always present in a single instance, including multiset $\boldsymbol{A}$ itself, which is the maximal multiset for family $\boldsymbol{P}(\boldsymbol{A})$, set $\operatorname{Supp} \boldsymbol{A}$, and the empty multiset $\varnothing$. Boolean $\boldsymbol{P}(\boldsymbol{A})$ is a set of submultisets of multiset $\boldsymbol{A}$.

Let us call a family of all possible submultisets of multiset $\boldsymbol{A}$ over domain $X$ (power multiset) a multiboolean of multiset $\boldsymbol{A}$ and denote it as $\boldsymbol{Q}(\boldsymbol{A})$. Multiboolean $\boldsymbol{Q}(\boldsymbol{A})$, in contrast to Boolean $\boldsymbol{P}(\boldsymbol{A})$, is a multiset in which several identical submultisets of multiset $\boldsymbol{A}$ are allowed. Support $\operatorname{Supp} \boldsymbol{Q}(\boldsymbol{A})$ of multiboolean $\boldsymbol{Q}(\boldsymbol{A})$ is Boolean $\boldsymbol{P}(\boldsymbol{A})$ of multiset $\boldsymbol{A}$. If $A \subseteq \boldsymbol{A}$, then $P(\boldsymbol{A}) \subseteq \boldsymbol{P}(\boldsymbol{A}) \subseteq$ $\boldsymbol{Q}(\boldsymbol{A})$. Some of the elements of Booleans $P(\boldsymbol{A}), \boldsymbol{P}(\boldsymbol{A})$, and multiboolean $\boldsymbol{Q}(\boldsymbol{A})$ themselves are sub(multi)sets of other (multi)sets of these families.

The cardinality of a family of sets and multisets $P(\boldsymbol{A}), \boldsymbol{P}(\boldsymbol{A})$, and $\boldsymbol{Q}(\boldsymbol{A})$ is determined by the total number of its elements (respectively, subsets of set $A$ and submultisets of multiset $\boldsymbol{A}$ ).

As an example, the cardinality of Boolean $P(A)$, the family of all subsets of the $n$-element set $A=\left\{x_{1}, x_{2}\right.$, $\left.\ldots, x_{n}\right\}$ is equal to card $P(A)=|P(A)|=2^{n}=2^{|A|}=2^{\operatorname{card} A}$.

The cardinality of Boolean $\boldsymbol{P}(\boldsymbol{A})$, the family of all various subsets of the $n$-dimensional multiset $\boldsymbol{A}=$ $\left\{k_{A}\left(x_{1}\right) \circ x_{1}, k_{A}\left(x_{2}\right) \circ x_{2}, \ldots, k_{A}\left(x_{i}\right) \circ x_{n}\right\}$ over a finite $n$ element set $X=\left\{x_{1}, x_{2}, \ldots, x_{n}\right\}$ is equal to $\operatorname{card} \boldsymbol{P}(\boldsymbol{A})=$ $|\boldsymbol{P}(\boldsymbol{A})|=\left(1+k_{A}\left(x_{1}\right)\right) \cdot\left(1+k_{A}\left(x_{2}\right)\right) \cdot \ldots \cdot\left(1+k_{A}\left(x_{n}\right)\right)=$ $\prod_{i=1}^{n}\left(1+k_{A}\left(x_{i}\right)\right)$.

The cardinality of multiboolean $Q(A)$, the family of all possible submultisets of an $n$-dimensional multiset $\boldsymbol{A}=\left\{k_{A_{1}}\left(x_{1}\right) \circ x_{1}, k_{A_{2}}\left(x_{2}\right) \circ x_{2}, \ldots, k_{A_{n}}\left(x_{i}\right) \circ x_{n}\right\}$ over the finite $n$-element set $X=\left\{x_{1}, x_{2}, \ldots, x_{n}\right\}$, is equal to $\operatorname{card} \boldsymbol{Q}(\boldsymbol{A})=|\boldsymbol{Q}(\boldsymbol{A})|=2^{m}=2^{|\boldsymbol{A}|}=2^{\operatorname{card} \boldsymbol{A}}$.

The capacity or volume of a family of sets and multisets is the sum of cardinalities of (multi)sets that make up this family. Then, respectively, the volume of Boolean $P(A)$ of set $A$ is $\operatorname{vol} P(A)=\|P(A)\|=$ $\sum_{A_{j} \in P(A)}\left|A_{j}\right|=\sum_{A_{j} \in P(A)} \sum_{x} \chi_{A_{j}}(x)$, the volume of Boolean $\boldsymbol{P}(\boldsymbol{A})$ of multiset $\boldsymbol{A}$ over set $X$ is $\operatorname{vol} \boldsymbol{P}(\boldsymbol{A})=$ $\|\boldsymbol{P}(\boldsymbol{A})\|=\sum_{\boldsymbol{A}_{j} \in \boldsymbol{P}(\boldsymbol{A})}\left|\boldsymbol{A}_{j}\right|=\sum_{\boldsymbol{A}_{j} \in \boldsymbol{P}(\boldsymbol{A})} \mid \sum_{x} k_{\boldsymbol{A}_{j}}(x)$, and the volume of multiboolean $\boldsymbol{Q}(\boldsymbol{A})$ of multiset $\boldsymbol{A}$ over set $X$ is $\operatorname{vol} \boldsymbol{Q}(\boldsymbol{A})=\|\boldsymbol{Q}(\boldsymbol{A})\|=\sum_{\boldsymbol{A}_{j} \in \boldsymbol{Q}(\boldsymbol{A})}\left|\boldsymbol{A}_{j}\right|=$ $\sum_{A_{j} \in Q(A)} \sum_{x} k_{A_{j}}(x)$.

Thus, Boolean $P(A)$ of set $A=\{a, b, c\}$ consists of sets $\varnothing,\{a\},\{b\},\{c\},\{a, b\},\{a, c\},\{b, c\}$, and $\{a, b, c\}$. The cardinality of Boolean $P(A)$ is equal to $|P(A)|=2^{3}=$ 8 , the volume $\|P(A)\|=12$.

Boolean $\boldsymbol{P}(\boldsymbol{A})$ of multiset $\boldsymbol{A}=\{3 \circ a, 1 \circ b, 2 \circ c\}$ over set $A=\{a, b, c\}$ includes multisets $\{0 \circ a, 0 \circ b, 0 \circ c\}=$ $\varnothing,\{1 \circ a, 0 \circ b, 0 \circ c\}=\{a\},\{0 \circ a, 1 \circ b, 0 \circ c\}=\{b\},\{0 \circ$ $a, 0 \circ b, 1 \circ c\}=\{c\},\{1 \circ a, 1 \circ b, 0 \circ c\}=\{a, b\},\{1 \circ a, 0 \circ$ $b, 1 \circ c\}=\{a, c\},\{0 \circ a, 1 \circ b, 1 \circ c\}=\{b, c\},\{2 \circ a, 0 \circ$ $b, 0 \circ c\},\{0 \circ a, 0 \circ b, 2 \circ c\},\{1 \circ a, 1 \circ b, 1 \circ c\}=\{a, b$, $c\}=\operatorname{Supp} A,\{3 \circ a, 0 \circ b, 0 \circ c\},\{2 \circ a, 1 \circ b, 0 \circ c\},\{2 \circ a$, $0 \circ b, 1 \circ c\},\{1 \circ a, 0 \circ b, 2 \circ c\},\{0 \circ a, 1 \circ b, 2 \circ c\},\{3 \circ$ $a, 1 \circ b, 0 \circ c\},\{3 \circ a, 0 \circ b, 1 \circ c\},\{2 \circ a, 0 \circ b, 2 \circ c\},\{2 \circ$ $a, 1 \circ b, 1 \circ c\},\{1 \circ a, 1 \circ b, 2 \circ c\},\{3 \circ a, 0 \circ b, 2 \circ c\},\{3 \circ$ $a, 1 \circ b, 1 \circ c\},\{2 \circ a, 1 \circ b, 2 \circ c\}$, and $\{3 \circ a, 1 \circ b, 2 \circ$ $c\}=\boldsymbol{A}$. The cardinality of Boolean $\boldsymbol{P}(\boldsymbol{A})$ is equal to $|P(A)|=4 \cdot 2 \cdot 3=24$, the volume of $\|P(A)\|=72$. In particular, the cardinality of Boolean $P(\varnothing)=\{\varnothing\}$ of the empty (multi)set $\varnothing$ is equal to $|\{\varnothing\}|=2^{0}=1$, the volume capacity $\|\{\varnothing\}\|=0$.

Multiboolean $\boldsymbol{Q}(\boldsymbol{A})$ of multiset $\boldsymbol{A}=\{3 \circ a, 1 \circ b, 2 \circ c\}$ over set $A=\{a, b, c\}$ contains 1 multiset $\{0 \circ a, 0 \circ b, 0 \circ c\}=$ $\varnothing, 3$ multisets $\{1 \circ a, 0 \circ b, 0 \circ c\}=\{a\}, 1$ multiset $\{0 \circ$ $a, 1 \circ b, 0 \circ c\}=\{b\}, 2$ multisets $\{0 \circ a, 0 \circ b, 1 \circ c\}=$ $\{c\}, 3$ multisets $\{1 \circ a, 1 \circ b, 0 \circ c\}=\{a, b\}, 6$ multisets $\{1 \circ a, 0 \circ b, 1 \circ c\}=\{a, c\}, 2$ multisets $\{0 \circ a, 1 \circ b, 1 \circ$ $c\}=\{b, c\}, 3$ multisets $\{2 \circ a, 0 \circ b, 0 \circ c\}, 1$ multiset $\{0 \circ$ $a, 0 \circ b, 2 \circ c\}, 6$ multisets $\{1 \circ a, 1 \circ b, 1 \circ c\}=\{a, b, c\}=$ $\operatorname{Supp} A, 1$ multiset $\{3 \circ a, 0 \circ b, 0 \circ c\}, 3$ multisets $\{2 \circ$ $a, 1 \circ b, 0 \circ c\}, 6$ multisets $\{2 \circ a, 0 \circ b, 1 \circ c\}, 3$ multisets $\{1 \circ a, 0 \circ b, 2 \circ c\}, 1$ multiset $\{0 \circ a, 1 \circ b, 2 \circ c\}, 1$ multiset $(3 \circ a, 1 \circ b, 0 \circ c\}, 2$ multisets $\{3 \circ a, 0 \circ b, 1 \circ c\}$, 3 multisets $\{2 \circ a, 0 \circ b, 2 \circ c\}, 6$ multisets $\{2 \circ a, 1 \circ b$, $1 \circ c\}, 3$ multisets $\{1 \circ a, 1 \circ b, 2 \circ c\}, 1$ multiset $\{3 \circ a$, $0 \circ b, 2 \circ c\}, 2$ multisets $\{3 \circ a, 1 \circ b, 1 \circ c\}, 3$ multisets $\{2 \circ a, 1 \circ b, 2 \circ c\}$, and 1 multiset $\{3 \circ a, 1 \circ b, 2 \circ c\}=\boldsymbol{A}$. The cardinality of multiboolean $Q(A)$ is equal to $|Q(A)|=$ $2^{6}=64$, the volume $\|Q(A)\|=192$. 
For a family of nonempty multisets $\boldsymbol{A}_{1}, \ldots, \boldsymbol{A}_{r}$, their union $\bigcup_{s=1}^{r} A_{s}=\boldsymbol{A}$ is called covering of multiset $\boldsymbol{A}$, their intersection $\bigcap_{s=1}^{r} \boldsymbol{A}_{s}=\boldsymbol{A}$ is overlapping of multiset $\boldsymbol{A}$, and their sum $\sum_{s=1}^{r} \boldsymbol{A}_{s}=\boldsymbol{A}$ is decomposition of multiset $\boldsymbol{A}$. If the multisets are pairwise disjoint $\left(\boldsymbol{A}_{p} \bigcap \boldsymbol{A}_{q}=\varnothing\right.$, $p \neq q$ : such multisets are said to be disjoint $)$, then covering and decomposition of multiset $\boldsymbol{A}$ coincide and are called partition of multiset $\boldsymbol{A}$. Multisets $\boldsymbol{A}_{1}, \ldots, \boldsymbol{A}_{r}$ that form the covering, overlapping, decomposition, and partition of multiset $\boldsymbol{A}$ are called its blocks, and the number $r$ of blocks is the rank of covering, overlapping, decomposition, or partition. Covering of multiset $\boldsymbol{A}$ of rank $r$ is denoted as $\boldsymbol{C}^{r}(\boldsymbol{A})=\left\{\boldsymbol{A}_{1} ; \ldots ; \boldsymbol{A}_{s} ; \ldots ; \boldsymbol{A}_{r}\right\}$, overlapping of multiset $\boldsymbol{A}$ of $\operatorname{rank} \boldsymbol{r}$ as $\boldsymbol{I}^{r}(\boldsymbol{A})=\left\{\boldsymbol{A}_{1} ; \ldots ; \boldsymbol{A}_{s}\right.$; ...; $\left.\boldsymbol{A}_{r}\right\}$, decomposition of multiset $\boldsymbol{A}$ of $\operatorname{rank} \boldsymbol{r}$ as $\boldsymbol{D}^{r}(\boldsymbol{A})=$ $\left\{\boldsymbol{A}_{1} ; \ldots ; \boldsymbol{A}_{s} ; \ldots ; \boldsymbol{A}_{r}\right\}$, and partition of multiset $\boldsymbol{A}$ of rank $\boldsymbol{r}$ as $\boldsymbol{B}^{r}(\boldsymbol{A})=\left\{\boldsymbol{A}_{1} ; \ldots ; \boldsymbol{A}_{s} ; \ldots ; \boldsymbol{A}_{r}\right\}$, where blocks are separated by semicolons. If all blocks are submultisets of multiset $\boldsymbol{A}$, in this case one speaks of partition of multiset $\boldsymbol{A}$ into classes. For the cardinality and volume of covering $\boldsymbol{C}^{r}(\boldsymbol{A})$, overlapping $\boldsymbol{I}^{r}(\boldsymbol{A})$, decomposition $D^{r}(\boldsymbol{A})$, and partition $\boldsymbol{B}^{r}(\boldsymbol{A})$ of multiset $\boldsymbol{A}$, we have:

$$
\begin{gathered}
\left|\boldsymbol{C}^{\mid r}(\boldsymbol{A})\right|=\left|\boldsymbol{I}^{\mid r}(\boldsymbol{A})\right|=\left|\boldsymbol{D}^{\mid r}(\boldsymbol{A})\right|=\left|\boldsymbol{B}^{\mid r}(\boldsymbol{A})\right|=r, \\
\left\|\boldsymbol{C}^{r}(\boldsymbol{A})\right\| \geq|\boldsymbol{A}|, \quad\left\|\boldsymbol{f}^{r}(\boldsymbol{A})\right\| \geq|\boldsymbol{A}|, \\
\left\|\boldsymbol{D}^{\mid r}(\boldsymbol{A})\right\|=\left\|\boldsymbol{B}^{\mid r}(\boldsymbol{A})\right\|=|\boldsymbol{A}| .
\end{gathered}
$$

Coverings and partitions of a set and coverings, decomposition, and partitions of a multiset can be ordered and unordered, depending on the priority of their blocks.

As an example, a family of zerons $\left\{\left\{0 \circ x_{1}\right\} ; \ldots ;\{0 \circ\right.$ $\left.\left.x_{s}\right\} ; \ldots ;\left\{0 \circ x_{n}\right\}\right\}$ is partition $B^{n}(\varnothing)$ of an empty (multi)set $\varnothing$, a family of singletons $\left\{\left\{1 \circ x_{1}\right\} ; \ldots ;\left\{1 \circ x_{s}\right\}\right.$; $\left.\ldots ;\left\{1 \circ x_{n}\right\}\right\}$ is partition $B^{n}(X)$ of the set $X=\left\{x_{1}, \ldots, x_{n}\right\}$, a family of multitons $\left\{\left\{k_{A}\left(x_{1}\right) \circ x_{1}\right\} ; \ldots ;\left\{k_{A}\left(x_{s}\right) \circ x_{s}\right\} ; \ldots\right.$; $\left.\left\{k_{\boldsymbol{A}}\left(x_{n}\right) \circ x_{n}\right\}\right\}$ is partition $\boldsymbol{B}^{n}(\boldsymbol{A})$ of multiset $\boldsymbol{A}=\left\{k_{\boldsymbol{A}}\left(x_{1}\right)\right.$ $\left.\left.\circ x_{1}, \ldots, k_{A}\left(x_{S}\right) \circ x_{s}\right\}, \ldots, k_{A}\left(x_{n}\right) \circ x_{n}\right\}$ with the number of blocks $r=|X|=n$. Boolean $P(A)$ of set $A$ is covering $C^{r}(A)$ of the set $A$ with the number of blocks $r=|P(A)|=$ $2^{|A|}$. Boolean $\boldsymbol{P}(\boldsymbol{A})$ of the $n$-dimensional multiset $\boldsymbol{A}=$ $\left\{k_{A}\left(x_{1}\right) \circ x_{1}, \ldots, k_{A}\left(x_{n}\right) \circ x_{n}\right\}$ over the finite set $X=\left\{x_{1}, \ldots\right.$, $\left.x_{n}\right\}$ is covering $\boldsymbol{C}^{r}(\boldsymbol{A})$ of multiset $\boldsymbol{A}$ with the number of blocks $r=|\boldsymbol{P}(\boldsymbol{A})|=\prod_{i=1}^{n}\left(1+k_{\boldsymbol{A}}\left(x_{i}\right)\right)$.

A family of multisets over set $X=\left\{x_{1}, x_{2}, \ldots\right\}$ is called semiring $E$ of multisets if it contains an empty multiset $\varnothing$, intersection $\boldsymbol{A}_{i} \bigcap \boldsymbol{A}_{j}$ of multisets $\boldsymbol{A}_{i}, \boldsymbol{A}_{j} \in \boldsymbol{E}$, and every multiset $\boldsymbol{A} \in \boldsymbol{E}$ is representable as a finite union $\bigcup_{s=1}^{r} \boldsymbol{A}_{s}=\boldsymbol{A}$ of disjoint submultisets $\boldsymbol{A}_{s} \subset \boldsymbol{A}$; ring $\boldsymbol{K}$ of submultisets if it contains an empty multiset $\varnothing$, union $\boldsymbol{A}_{i} \bigcup \boldsymbol{A}_{j}, \operatorname{sum} \boldsymbol{A}_{i}+\boldsymbol{A}_{j}$, and difference $\boldsymbol{A}_{i}-\boldsymbol{A}_{j}$ of multisets $\boldsymbol{A}_{i}, \boldsymbol{A}_{j} \in \boldsymbol{K}$; algebra $\boldsymbol{S}$ of multisets if it is a ring that includes the maximal set, called a unit of algebra, and is closed in relation to finite unions, additions, and complements of multisets; $\sigma$-ring $\boldsymbol{K}_{\sigma}$ of multisets if it is a ring and contains a countable union $\bigcup_{s=1}^{\infty} \boldsymbol{A}_{s}$ and sum $\sum_{s=1}^{\infty} \boldsymbol{A}_{s}$ of multisets $\boldsymbol{A}_{s} \in \boldsymbol{K}_{\sigma}, \delta$-ring $\boldsymbol{K}_{\delta}$ of multisets if it is a ring and contains a countable intersection $\bigcap_{s=1}^{\infty} \boldsymbol{A}_{s}$ of multisets $\boldsymbol{A}_{s} \in \boldsymbol{K}_{\delta}, \sigma$-algebra $\boldsymbol{S}_{\sigma}$ of multisets if it is an $\sigma$-ring, contains a unit of algebra, and is closed in relation to countable unions, additions, and complements of multisets.

In particular, every ring of sets/multisets is a semiring of sets/multisets, and every $\sigma$-algebra of sets/multisets is a $\delta$-algebra of sets/multisets, and vice versa.

Boolean $\mathrm{P}(A)$ of set $A$ is the minimal algebra of subsets of set $A$, which is a unit of the algebra. The Boolean algebra $B(A)$ of subsets of set $A$ is $\sigma$-algebra of set $X$, which is a unit of the algebra, and family $P(A)$ is the support of the algebra. Boolean $\boldsymbol{P}(\boldsymbol{A})$ of the $n$ dimensional multiset $\boldsymbol{A}=\left\{k_{\boldsymbol{A}}\left(x_{1}\right) \circ x_{1}, \ldots, k_{\boldsymbol{A}}\left(x_{n}\right) \circ x_{n}\right\}$ over the finite set $X=\left\{x_{1}, \ldots, x_{n}\right\}$ is the minimal algebra of submultisets of multiset $\boldsymbol{A}$, which is a unit of algebra. The Boolean algebra $\boldsymbol{B}(\boldsymbol{A})$ of submultisets of multiset $\boldsymbol{A}=\left\{k_{A}\left(x_{1}\right) \circ x_{1}, k_{A}\left(x_{2}\right) \circ x_{2}, \ldots\right\}$ over the infinite set $X=$ $\left\{x_{1}, x_{2}, \ldots\right\}$ is $\sigma$-algebra of multiset $\boldsymbol{A}$, which is a unit of algebra.

When turning to sets, decomposition of the set will be impossible, and covering, partition, semiring, ring, and algebra of the multiset will turn into the covering, partition, semiring, ring, and algebra of the set, respectively.

A nonnegative real function of multiset $m: \boldsymbol{A} \rightarrow \mathbb{R}$, defined on family $\boldsymbol{A}$, is said to be a strongly additive or a strongly finitely additive measure if for any finite set of multisets $\boldsymbol{A}_{i} \in \boldsymbol{A}$ the following equality holds:

$$
m\left(\sum_{i=1}^{\infty} \boldsymbol{A}_{i}\right)=\sum_{i=1}^{n} m\left(\boldsymbol{A}_{i}\right),
$$

and a strongly $\sigma$-additive or a strongly countable additive measure if for any countable collection of multisets $\boldsymbol{A}_{i} \in \boldsymbol{A}$ the following equality holds:

$$
m\left(\sum_{i=1}^{\infty} \boldsymbol{A}_{i}\right)=\sum_{i=1}^{\infty} m\left(\boldsymbol{A}_{i}\right) .
$$

As family $\boldsymbol{A}$ we have semiring $\boldsymbol{E}$, ring $\boldsymbol{K}, \sigma$-ring $\boldsymbol{K}_{\sigma}$, or $\sigma$-algebra $\boldsymbol{S}_{\sigma}$ of submultisets $\boldsymbol{A}_{i}$ of some multiset $\boldsymbol{A}=$ $\left\{k_{A}\left(x_{1}\right) \circ x_{1}, k_{A}\left(x_{2}\right) \circ x_{2}, \ldots\right\}$ over set $X=\left\{x_{1}, x_{2}, \ldots\right\}$. Such a multiset $\boldsymbol{A}$ can be, for example, the maximal multiset $\boldsymbol{Z}=\left\{k_{Z}\left(x_{1}\right) \circ x_{1}, k_{Z}\left(x_{2}\right) \circ x_{2}, \ldots\right\}$, constant multiset $\boldsymbol{Z}_{[k]}=$ $\left\{k \circ x_{1}, k \circ x_{2}, \ldots\right\}$, or $n$-dimensional multiset $\boldsymbol{X}=$ 
$\left\{k_{X}\left(x_{1}\right) \circ x_{1}, \ldots, k_{X}\left(x_{n}\right) \circ x_{n}\right\}$. From the identity property of an empty multiset $\varnothing=\varnothing+\varnothing$ and equality (2) it follows that $m(\varnothing)=m(\varnothing+\varnothing)=2 m(\varnothing)$, and hence $m(\varnothing)=0$.

For any finite or countable partition of multiset $\boldsymbol{A}$, the sum of submultisets coincides with their union $\sum_{i=1}^{n} A_{i} \bigcup_{i=1}^{n} A_{i}$. Then, expressions (2) and (3) for disjoint multisets $\boldsymbol{A}_{i} \bigcap \boldsymbol{A}_{j}=\varnothing, i \neq j, \boldsymbol{A}_{i}, \boldsymbol{A}_{j} \in \boldsymbol{A}$ can be written as

$$
\begin{aligned}
& m\left(\bigcup_{i=1}^{n} \boldsymbol{A}_{i}\right)=\sum_{i=1}^{n} m\left(\boldsymbol{A}_{i}\right), \\
& m\left(\bigcup_{i=1}^{\infty} \boldsymbol{A}_{i}\right)=\sum_{i=1}^{\infty} m\left(\boldsymbol{A}_{i}\right),
\end{aligned}
$$

A multiset measure that satisfies condition (4) will be called weakly additive or weakly finitely additive, and a measure that satisfies condition (5) will be called weakly $\sigma$-additive or weakly countably additive.

The strongly additive multiset function is also weakly additive. The converse, generally speaking, is not true. The weak additivity (4), (5) of the multiset function coincides with the finite and countable additivity of the set function. For sets, the addition operation is not feasible and there is no strong additivity of the function. Thus, the additivity of multiset functions is more diverse than the additivity of set functions.

The measure $m$ of a multiset also has the properties of monotonicity $m(\boldsymbol{A}) \leq m(\boldsymbol{B}) \Leftrightarrow \boldsymbol{A} \subseteq \boldsymbol{B}$; symmetry $m(\boldsymbol{A})+m(\overline{\boldsymbol{A}})=m(\boldsymbol{Z})$; continuity $\lim _{i \rightarrow \infty} m\left(\boldsymbol{A}_{i}\right)=$ $m\left(\lim _{i \rightarrow \infty} \boldsymbol{A}_{i}\right)$; and elasticity $m(b \cdot \boldsymbol{A})=b m(\boldsymbol{A})$, where $b \geq 1$ is an integer. Let the measure of singleton $\left\{x_{i}\right\}$ be equal to $m\left(\left\{x_{i}\right\}\right)=w_{i}, 0 \leq w_{i}<\infty$. Then, according to equalities (2) and (3), the function defined on the family of multisets $\boldsymbol{A}$

$$
\begin{gathered}
m(\boldsymbol{A})=m\left(\sum_{i} \boldsymbol{A}_{i}\right)=\sum_{i} m(\boldsymbol{A}) \\
=\sum_{i}\left[k_{\boldsymbol{A}}\left(x_{i}\right) m\left(\left\{x_{i}\right\}\right)\right]=\sum_{i} w_{i} k_{\boldsymbol{A}}\left(x_{i}\right)
\end{gathered}
$$

is strongly additive or, respectively, strongly $\sigma$-additive measure of a multiset. If in (6) all the numbers are $w_{i}=1$, we obtain: $m(\boldsymbol{A})=\sum_{i} k_{\boldsymbol{A}}\left(x_{i}\right)=|\boldsymbol{A}|$. Thus, the cardinality of a multiset is a special case of its measure.

Let us note the important properties of the multiset measure that are due to its strong additivity and resemble the properties of the cardinality of a multiset:

$$
\begin{gathered}
m(\boldsymbol{A}+\boldsymbol{B})=m(\boldsymbol{A})+m(\boldsymbol{B}) \\
=m(\boldsymbol{A} \bigcup \boldsymbol{B})+m(\boldsymbol{A} \bigcap \boldsymbol{B}), \\
m(\boldsymbol{A}-\boldsymbol{B})=m(\boldsymbol{A})-m(\boldsymbol{A} \bigcap \boldsymbol{B}),
\end{gathered}
$$

$$
\begin{gathered}
m(\boldsymbol{A} \Delta \boldsymbol{B})=m(\boldsymbol{A}-\boldsymbol{B})+m(\boldsymbol{B}-\boldsymbol{A}) \\
=m(\boldsymbol{A} \bigcup \boldsymbol{B})-m(\boldsymbol{A} \bigcap \boldsymbol{B}), \\
m\left(\bigcap_{i=1}^{n} \boldsymbol{A}_{i}\right) \leq m\left(\bigcup_{i=1}^{n} \boldsymbol{A}_{i}\right) \leq \sum_{i=1}^{n} m\left(\boldsymbol{A}_{i}\right) .
\end{gathered}
$$

The space of multisets with a measure is defined to be a pair $\left(\boldsymbol{S}_{\sigma}(\boldsymbol{Z}), m\right)$, where $\boldsymbol{S}_{\sigma}(\boldsymbol{Z})$ is $\sigma$-algebra over a maximal multiset $\boldsymbol{Z}, m$ is a $\sigma$-finite measure defined on $\sigma$-ring $\boldsymbol{K}_{\sigma}$ of submultisets $\boldsymbol{A}_{i} \subseteq \boldsymbol{Z}$ that form the decomposition of multiset $\boldsymbol{Z}=\sum_{i=1}^{\infty} \boldsymbol{A}_{i}$. The sum, union, and intersection of a finite number of measurable multisets, the difference and the symmetric difference of two measurable multisets, and the reproduction of a measurable multiset are measurable multisets. Necessary and sufficient conditions for the measurability of a multiset are formulated as follows $[6,7]$. Multiset $\boldsymbol{A}$ belonging to $\boldsymbol{\sigma}$-ring $\boldsymbol{K}_{\sigma}$ is measurable if and only if for any $\varepsilon>0$ there exists a measurable multiset $\boldsymbol{B} \in \boldsymbol{K}_{\sigma}$ such that inequality $m(\boldsymbol{A} \Delta \boldsymbol{B})<\varepsilon$ is satisfied.

\section{METRIC SPACES OF MULTISETS}

A set in which the concept of proximity of elements is defined in some way is usually called a space and its elements are called points of space. A nonnegative real function $d_{X}$ defined on the direct product $X \times X$ of set $X$ is called a metric on set $X$ if for any elements the following axioms are satisfied: axioms of $\left(1^{0}\right)$ symmetry $d_{X}(x, y)=d_{X}(y, x) ;\left(2^{0}\right)$ identity $d_{X}(x, y)=0 \Leftrightarrow x=y$; and $\left(3^{0}\right)$ triangle $d_{X}(x, y) \leq d_{X}(x, z)+d_{X}(z, y)$. The numerical value of function $d_{X}(x, y)$ is called the distance between elements $x$ and $y$ of set $X$. Requirements $\left(1^{0}\right)-\left(3^{0}\right)$ imply the nonnegativity of metric $d_{X}(x, y) \geq$ 0 . Set $X$ with metric $d_{X}$ defined on it is called a metric space and is denoted by $\left(X, d_{X}\right)$.

A metric space $X$ is said to be metrically convex or $d$-convex if for any noncoinciding elements $x, y \in X, x \neq$ $y$ there exists a distinct element $z \in X, z \neq x, z \neq y$ such that the triangle inequality $\left(3^{0}\right)$ becomes equality $d_{X}(x$, $y)=d_{X}(x, z)+d_{X}(z, y)$. Metric $d_{X}$ that satisfies this condition will be called $d$-convex. The property of the metric convexity of the space $\left(X, d_{X}\right)$ is the spatial ana$\log$ of the ternary relation $[x, z, y]$ "element $z$ lies between elements $x$ and $y$," which in an arbitrary partially ordered set is given by condition $\inf (x, y) \leq z \leq$ $\sup (x, y)$.

The relative positions of the elements of set $X$ are also estimated using other indices of the proximity of the elements, which are based on the incomplete axiomatics of the metric space. The nonnegative real function $d_{X}$ defined on the direct product $X \times X$, which satisfies the symmetry axiom $\left(1^{0}\right)$ and the coincidence condition $\left(4^{0}\right) d_{X}(x, x)=0$ for all $x \in X$, is called a quasimetric, and the one that satisfies the triangle axiom 
$\left(3^{0}\right)$ is called a pseudometric on set $X$. The corresponding spaces $\left(X, d_{X}\right)$ are called quasimetric and pseudometric. In the quasi- and pseudometric spaces the identity axiom $\left(2^{0}\right)$ for function $d_{X}$ is not satisfied, that is, condition $d_{X}(x, y)=0$ does not, in general, imply the equality of elements $x$ and $y$. The coincidence condition $\left(4^{0}\right)$ is weaker than the identity axiom $\left(2^{0}\right)$. Therefore, every metric is also a quasimetric and a pseudometric. The converse is not true in the general case.

A nonnegative real function $d_{X}$ defined on the direct product $X \times X$ and that satisfies the symmetry $\left(1^{0}\right)$ and identity $\left(2^{0}\right)$ axioms is called a symmetric on set $X$, and set $X$ with the symmetric $d_{X}$ is called a proximity space. Symmetric $d_{X}$ that satisfies the inequality $\left(5^{0}\right) d_{X}(x, y) \leq \max _{z \in X}\left[d_{X}(x, z), d_{X}(z, y)\right]$ for any elements of set $X$ is called the ultrametric on set $X$ or the ultrametric distance between elements $x$ and $y$. It is easy to verify that the triangle axiom $\left(3^{0}\right)$ also holds for the ultrametric. Thus, ultrametric $d_{X}$ satisfies the axiomatics of the metric space, and space $\left(X, d_{X}\right)$ with ultrametric $d_{X}$ is metric. The inequality $\left(5^{0}\right)$ is stronger than the triangle inequality $\left(3^{\circ}\right)$. Therefore, any ultrametric is also a metric. The converse is not true in the general case.

Let us consider possible approaches to the formation of metric spaces $\left(\boldsymbol{A}, d_{\boldsymbol{A}}\right)$ on families of $\boldsymbol{A}$ sets and $\boldsymbol{A}$ multisets. Any set $A \subseteq X$ and multiset $\boldsymbol{A} \subseteq Z$ over set $X=\left\{x_{1}, \ldots, x_{n}\right\}$ can be associated with an integer vector $\mathbf{y}=\left(y_{1}, \ldots, y_{n}\right)$ whose components are given by, respectively, as $y_{i}=\chi_{A}\left(x_{i}\right)=0,1$ for set $A$ and $y_{i}=k_{A}\left(x_{i}\right)=0$, $1,2, \ldots$ for multiset $\boldsymbol{A}, x_{i} \in X$. As an example, vector $(1,1,1,1,1,1,1,1)$ corresponds to set $X=\{a, b, c, d, e, f$, $g$, vector $(0,0,0,0,0,0,0)$ corresponds to the empty (multi)set $\varnothing$, vector $(1,0,0,0,0,0,0,0)$ corresponds to singleton $\{a\}$, vector $(0,2,0,0,0,0,0,0)$ corresponds to multiton $\{2 \circ b\}$, vector $(1,1,1,0,0,0,0,0)$ corresponds to set $A=\{a, b, c\}$, and vector $(3,1,2,0,0,0,0)$ corresponds to multiset $\boldsymbol{A}=\{3 \circ a, 1 \circ b, 2 \circ c\}$.

We use an analogy with known types of metric spaces: vector spaces $l_{p}^{n}$ and spaces of bounded numerical sequences $l_{p}[1]$.

Boolean $\boldsymbol{P}(\boldsymbol{X})$ of various submultisets of an $n$-dimensional multiset $X=\left\{k_{X}\left(x_{1}\right) \circ x_{1}, \ldots, k_{X}\left(x_{n}\right) \circ x_{n}\right\}$ over the finite set $X=\left\{x_{1}, \ldots, x_{n}\right\}$ is equivalent to set $\mathbb{R}^{n}$ of $n$-dimensional vectors $\mathbf{y}_{s}=\left(y_{s^{1}}, \ldots y_{s^{n}}\right)$ with real components $y_{s^{i}}=k_{A_{s}}\left(x_{i}\right)$ and forms a metric space of finite multisets [19]:

$$
\begin{gathered}
\boldsymbol{P}_{1}=\left(\boldsymbol{P}(\boldsymbol{X}), d_{\boldsymbol{P}_{1}}\right) \text { with the Hamming-type metric } \\
d_{\boldsymbol{P}_{1}}(\boldsymbol{A}, \boldsymbol{B})=\sum_{i=1}^{n}\left|k_{\boldsymbol{A}}\left(x_{i}\right)-k_{\boldsymbol{B}}\left(x_{i}\right)\right|
\end{gathered}
$$

$\boldsymbol{P}_{2}=\left(\boldsymbol{P}(\boldsymbol{X}), d_{\boldsymbol{P}_{2}}\right)$ with the Euclidean-type metric

$$
d_{\boldsymbol{P}_{2}}(\boldsymbol{A}, \boldsymbol{B})=\left[\sum_{i=1}^{n}\left|k_{\boldsymbol{A}}\left(x_{i}\right)-k_{\boldsymbol{B}}\left(x_{i}\right)\right|^{2}\right]^{1 / 2} ;
$$

$\boldsymbol{P}_{p}=\left(\boldsymbol{P}(\boldsymbol{X}), d_{\boldsymbol{P}_{p}}\right)$ with the Minkowski-type metric

$$
d_{\boldsymbol{P}_{p}}(\boldsymbol{A}, \boldsymbol{B})=\left[\sum_{i=1}^{n}\left|k_{A}\left(x_{i}\right)-k_{\boldsymbol{B}}\left(x_{i}\right)\right|^{p}\right]^{1 / p} ;
$$

$\boldsymbol{P}_{\infty}=\left(\boldsymbol{P}(\boldsymbol{X}), d_{\boldsymbol{P}_{\infty}}\right)$ with the Chebyshev-type metric

$$
d_{\boldsymbol{P}_{\infty}}(\boldsymbol{A}, \boldsymbol{B})=\max _{i}\left|k_{\boldsymbol{A}}\left(x_{i}\right)-k_{\boldsymbol{B}}\left(x_{i}\right)\right| .
$$

Here, $p \geq 1$ is an integer.

$\sigma$-algebra $\boldsymbol{S}_{\sigma}(\boldsymbol{X})$ of different submultisets of multiset $X=\left\{k_{X}\left(x_{1}\right) \circ x_{1}, k_{X}\left(x_{2}\right) \circ x_{2}, \ldots\right\}$, over an arbitrary set $X=\left\{x_{1}, x_{2}, \ldots\right\}$, where any submultiset $\boldsymbol{A}_{s} \subseteq \boldsymbol{X}$ satisfies the boundedness condition $k_{A_{s}}\left(x_{i}\right) \leq c_{s}<\infty$ or $\sum_{i=1}^{\infty}\left(k_{A_{s}}\left(x_{i}\right)\right)^{p} \leq c^{p}<\infty$ for all integers $p \geq 1$, is equivalent to set $\mathbb{R}^{N}$ of bounded numerical sequences $Y_{s}=$ $\left\{y_{s_{i}}\right\}=\left\{y_{s_{1}}, y_{s_{2}}, \ldots\right\}$ with real terms $y_{s_{i}}=k_{A_{s}}\left(x_{i}\right)$ that satisfies the condition $\left|y_{s_{i}}\right| \leq c_{s}<\infty$ or $\sum_{i=1}^{\infty}\left|y_{s_{i}}\right|^{p} \leq c^{p}<\infty$ for all integers $p \geq 1$ and forms a metric space of bounded multisets:

$$
\begin{gathered}
\boldsymbol{S}_{1}=\left(\boldsymbol{S}_{\sigma}(\boldsymbol{X}), d_{\boldsymbol{S}_{1}}\right) \text { with the Hamming-type metric } \\
d_{\boldsymbol{S}_{1}}(\boldsymbol{A}, \boldsymbol{B})=\sum_{i=1}^{\infty}\left|k_{\boldsymbol{A}}\left(x_{i}\right)-k_{\boldsymbol{B}}\left(x_{i}\right)\right| ; \\
\boldsymbol{S}_{2}=\left(\boldsymbol{S}_{\sigma}(\boldsymbol{X}), d_{\boldsymbol{S}_{2}}\right) \text { with the Euclidean-type metric } \\
d_{\boldsymbol{S}_{2}}(\boldsymbol{A}, \boldsymbol{B})=\left[\sum_{i=1}^{\infty}\left|k_{\boldsymbol{A}}\left(x_{i}\right)-k_{\boldsymbol{B}}\left(x_{i}\right)\right|^{2}\right]^{1 / 2} ;
\end{gathered}
$$

$\boldsymbol{S}_{p}=\left(\boldsymbol{S}_{\sigma}(\boldsymbol{X}), d_{\boldsymbol{S}_{p}}\right)$ with the Minkowski-type metric

$$
d_{S_{p}}(\boldsymbol{A}, \boldsymbol{B})=\left[\sum_{i=1}^{\infty}\left|k_{A}\left(x_{i}\right)-k_{\boldsymbol{B}}\left(x_{i}\right)\right|^{p}\right]^{1 / p} ;
$$

$\boldsymbol{S}_{\infty}=\left(\boldsymbol{S}_{\sigma}(\boldsymbol{X}), d_{\boldsymbol{S}_{\infty}}\right)$ with the Chebyshev-type metric

$$
d_{S_{\infty}}(\boldsymbol{A}, \boldsymbol{B})=\sup _{i}\left|k_{\boldsymbol{A}}\left(x_{i}\right)-k_{\boldsymbol{B}}\left(x_{i}\right)\right| \text {. }
$$

Let us note that expressions (9)-(11) and (13)(15) comprise the cardinality $|\boldsymbol{A} \Delta \boldsymbol{B}|=$ $\sum_{i}\left|k_{A}\left(x_{i}\right)-k_{B}\left(x_{i}\right)\right|$ of the symmetric difference of multisets $\boldsymbol{A}$ and $\boldsymbol{B}$, which plays the role of the metric. Replacing the multiplicity function $k_{A}(x)$ by the characteristic function $\chi_{A}(x)$ in formulas (9)-(16) we obtain definitions of metric spaces of finite and bounded sets.

The space $\left(\boldsymbol{S}_{\sigma}(\boldsymbol{Z}), m\right)$ of multisets with a measure, where multisets $\boldsymbol{A}_{s} \subseteq \boldsymbol{Z}$ that comprise the $\sigma$-algebra $\boldsymbol{S}_{\sigma}(\boldsymbol{Z})$ of the maximal multiset $\boldsymbol{Z}=\left\{k_{\boldsymbol{Z}}\left(x_{1}\right) \circ x_{1}, k_{Z}\left(x_{2}\right) \circ\right.$ $\left.x_{2}, \ldots\right\}$ over set $X=\left\{x_{1}, x_{2}, \ldots\right\}$ are measurable and measure $m$ of the multiset is strongly $\sigma$-additive and com- 
pletely $\sigma$-finite, forms the metric spaces of measurable multisets $\boldsymbol{Z}_{q p}=\left(\boldsymbol{S}_{\sigma}(\boldsymbol{Z}), d_{Z_{q p}}\right)$, where $q=1,2,3,4, p \geq 1$ is an integer, with the functions

$$
\begin{gathered}
d_{Z_{1 p}}(\boldsymbol{A}, \boldsymbol{B})=[m(\boldsymbol{A} \Delta \boldsymbol{B})]^{1 / p}, \\
d_{Z_{2 p}}(\boldsymbol{A}, \boldsymbol{B})=[m(\boldsymbol{A} \Delta \boldsymbol{B}) / m(\boldsymbol{Z})]^{1 / p}, \\
d_{Z_{3 p}}(\boldsymbol{A}, \boldsymbol{B})=[m(\boldsymbol{A} \Delta \boldsymbol{B}) / m(\boldsymbol{A} \bigcup \boldsymbol{B})]^{1 / p}, \\
d_{Z_{4 p}}(\boldsymbol{A}, \boldsymbol{B})=[m(\boldsymbol{A} \Delta \boldsymbol{B}) / m(\boldsymbol{A}+\boldsymbol{B})]^{1 / p} .
\end{gathered}
$$

Functions $d_{Z_{3 p}}$ and $d_{Z_{4 p}}$ are not defined for $\boldsymbol{A}=\boldsymbol{B}=$ $\varnothing$; therefore, by definition, we take $d_{Z_{3 p}}(\varnothing, \varnothing)=$ $d_{Z_{4_{p}}}(\varnothing, \varnothing)=0$. Function $d_{Z_{1 p}}$ defines mapping $d_{Z_{1 p}}$ : $\boldsymbol{S}_{\sigma} \times \boldsymbol{S}_{\sigma} \rightarrow \mathbb{R}_{+}$, and functions $d_{Z_{2 p}}, d_{Z_{3 p}}$, and $d_{Z_{4 p}}$ define mapping $d_{Z_{q p}}: \boldsymbol{S}_{\sigma} \times \boldsymbol{S}_{\sigma} \rightarrow \mathbb{R}_{01}=[0,1]$. Functions $d_{Z_{q p}}(\boldsymbol{A}, \boldsymbol{B}), q=1,2,3$ are pseudometrics that satisfy the symmetry $\left(1^{0}\right)$ and triangle $\left(3^{0}\right)$ axioms and the coincidence condition $\left(4^{0}\right)$, and function $d_{Z_{4 p}}(\boldsymbol{A}, \boldsymbol{B})$ is a quasimetric that satisfies the symmetry axiom $\left(1^{0}\right)$ and the coincidence condition $\left(4^{0}\right)$. In the general case, condition $m(\boldsymbol{A} \Delta \boldsymbol{B})=0$ does not imply that $\boldsymbol{A}=\boldsymbol{B}$. For multisets that differ by a multiset of measure zero, condition $m(\boldsymbol{A} \Delta \boldsymbol{B})=0$ implies the $m$-equality of such multisets $\boldsymbol{A}={ }_{m} \boldsymbol{B}$, and then for functions $d_{Z_{q p}}(\boldsymbol{A}, \boldsymbol{B})$, $q=1,2,3,4$, the identity axiom $\left(2^{0}\right)$ also holds. Functions $d_{Z_{1 p}}, d_{Z_{2 p}}$, and $d_{Z_{3 p}}$ become metrics, and function $d_{Z_{4 p}}$ is symmetric almost everywhere on $\sigma$-algebra $S_{\sigma}(Z)$ of measurable multisets. The proofs of these statements for pseudometrics were given in $[6,7]$, and for a quasimetric, in the appendix.

Let us call pseudometric $d_{Z_{1 p}}(\boldsymbol{A}, \boldsymbol{B})$ on space $\left(\boldsymbol{S}_{\sigma}(Z), m\right)$ of measurable multisets general, pseudometric $d_{Z_{2 p}}(\boldsymbol{A}, \boldsymbol{B})$ completely averaged, pseudometric $d_{Z_{3 p}}(A, B)$ locally averaged, and quasimetric $d_{Z_{4 p}}(A, B)$ averaged. The completely averaged pseudometric $d_{Z_{2 p}}(\boldsymbol{A}, \boldsymbol{B})$ characterizes the proximity of two multisets $\boldsymbol{A}$ and $\boldsymbol{B}$, referred to the maximal possible distance in the original space. The locally averaged pseudometric $d_{Z_{3 p}}(\boldsymbol{A}, \boldsymbol{B})$ characterizes the proximity of two multisets $\boldsymbol{A}$ and $\boldsymbol{B}$, referred to the joint "common part" of these two multisets in the original space. The averaged quasimetric $d_{Z_{4 p}}(\boldsymbol{A}, \boldsymbol{B})$ characterizes the proximity of two multisets $\boldsymbol{A}$ and $\boldsymbol{B}$, referred to the maximal possible "common part" of these two multisets in the original space.

The basic pseudometric $d_{Z_{1 p}}(\boldsymbol{A}, \boldsymbol{B})=[m(\boldsymbol{A} \Delta \boldsymbol{B})]^{1 / p}$ and the completely averaged pseudometric $d_{Z_{2 p}}(\boldsymbol{A}, \boldsymbol{B})=$ $[m(\boldsymbol{A} \Delta \boldsymbol{B}) / m(\boldsymbol{Z})]^{1 / p}$ are continuous, uniformly continuous, and equicontinuous functions, and the locally averaged pseudometric $d_{Z_{3 p}}(\boldsymbol{A}, \boldsymbol{B})=[m(\boldsymbol{A} \Delta \boldsymbol{B}) / m(\boldsymbol{A} \bigcup \boldsymbol{B})]^{1 / p}$ and the averaged quasimetric $d_{Z_{4 p}}(\boldsymbol{A}, \boldsymbol{B})=[m(\boldsymbol{A} \Delta \boldsymbol{B})=$ $[m(\boldsymbol{A} \Delta \boldsymbol{B})] / m(\boldsymbol{A}+\boldsymbol{B})]^{1 / p}$ are piecewise continuous functions of their variables almost everywhere on the corresponding metric space for any number $p[6,7]$. Pseudometrics $d_{Z_{q p}}(\boldsymbol{A}, \boldsymbol{B}), q=1,2,3$ and quasimetrics $d_{Z_{4 p}}(\boldsymbol{A}, \boldsymbol{B})$ are not similar to any metrics of other metric spaces, in particular, to Minkowski-type metrics (11), (15). When $w_{i}=1$ for all $i$ in formula (6), the general pseudometric $d_{Z_{11}}(\boldsymbol{A}, \boldsymbol{B})=|\boldsymbol{A} \Delta \boldsymbol{B}|$ coincides with the Hamming-type metrics (9), (13).

The metric spaces of multisets have the following properties [6, 7]. The spaces $\boldsymbol{P}_{p}, \boldsymbol{P}_{\infty}$ of finite multisets are metrically convex, $l_{p}^{n}$ - and $l_{p}$-embeddable, everywhere dense, separable, incomplete, noncompact, but relatively compact. The spaces $\boldsymbol{S}_{p}, \boldsymbol{S}_{\infty}$ of bounded multisets are metrically convex, separable, complete, noncompact, but locally compact. The spaces $Z_{1 p}=$ $\left(\boldsymbol{S}_{\sigma}(\boldsymbol{Z}), d_{Z_{1 p}}\right), \boldsymbol{Z}_{2 p}=\left(\boldsymbol{S}_{\sigma}(\boldsymbol{Z}), d_{Z_{2 p}}\right)$ of measurable multisets are homeomorphic, complete, and separable. The spaces $\boldsymbol{Z}_{11}=\left(\boldsymbol{S}_{\sigma}(\boldsymbol{Z}), d_{Z_{11}}\right), \boldsymbol{Z}_{21}=\left(\boldsymbol{S}_{\sigma}(\boldsymbol{Z}), d_{Z_{21}}\right), \boldsymbol{Z}_{31}=$ $\left(\boldsymbol{S}_{\sigma}(\boldsymbol{Z}), d_{Z_{31}}\right)$ of measurable multisets are metrically convex, and, in addition, the spaces $\boldsymbol{Z}_{11}$ and $\boldsymbol{Z}_{21}$ are isometric to the space of bounded numerical sequences $l_{1}$.

When the multiplicity function $k_{A}(x)$ is replaced by the characteristic function $\chi_{A}(x)$, the metric spaces of measurable multisets are transformed into the metric spaces of measurable sets that have many similar properties. The general metric $d_{X_{11}}(A, B)=m(A \Delta B)$ or $d_{X_{11}}(A, B)=|A \Delta B|$ is called the Fréchet-NikodymAronshayan distance or a metric of measure. The locally averaged metric $d_{X_{31}}(A, B)=$ $m(A \Delta B) / m(A \bigcup B)$ is called the Steinhaus distance and $d_{X_{31}}(A, B)=|A \Delta B| / \mid A \bigcup B$ is called the biotopic distance [1-3]. The spaces of measurable sets and multisets with metrics of the form (17)-(20) were first introduced in our papers [8-10].

\section{REPRESENTATION AND COMPARISON OF MULTI-ATTRIBUTE OBJECTS}

Let us discuss possible ways of representing and comparing objects that are present in several differing instances (versions or copies) and are described by many quantitative and qualitative attributes $[12,15]$.

Let us first consider the situation where the available objects $A_{1}, \ldots, A_{m}$ are in single instances and are characterized by attributes $K_{1}, \ldots, K_{n}$ with numerical and/or verbal scales of estimates $X_{l}=\left\{x_{l}^{1}, \ldots, x_{l}^{h_{l}}\right\}, l=$ $1, \ldots, n$. Traditionally, each object $A_{i}, i=1, \ldots, m$ is associated with a vector or tuple $\boldsymbol{x}_{i}=\left(x_{i_{1}}^{e_{1}}, \ldots, x_{i_{n}}^{e_{n}}\right)$, 
where $x_{i_{l}}^{e_{l}}$ is one of the gradations on scale $X_{l}$ of attribute $K_{l}$. Vector/tuple $x_{i}$ is a point of the $n$-dimensional space $X=X_{1} \times \ldots \times X_{n}$ formed by the scales of estimates of attributes $K_{1}, \ldots, K_{n}$. The collection of multi-attribute objects $A_{1}, \ldots, A_{m}$ and their attributes can also be represented by the table Objects-Attributes $\mathrm{F}=\left\|x_{i_{l}}\right\|_{m \times n}$. Rows of matrix F correspond to objects, columns correspond to attributes, and elements $x_{i,}$ of the matrix are values of components $x_{i_{l}}^{e_{l}}$ of vectors/tuples that specify objects.

The situation becomes significantly more complicated when the same multi-attribute object $A_{i}, i=1$, ..., $m$ is present in several instances (versions or copies) $A_{i}^{\langle s\rangle}, s=1, \ldots, t$, which differ in the attribute values $K_{1}$, $\ldots, K_{n}$. In such cases, the object $A_{i}$ will be associated not with one vector/tuple but a group of $t$ vectors/tuples $\left\{\boldsymbol{x}_{i}^{\langle 1\rangle}, \ldots, \boldsymbol{x}_{i}^{\langle t\rangle}\right\}$. Here, the vector/tuple $\boldsymbol{x}_{i}^{\langle s\rangle}=$ $\left(x_{i_{1}}^{\langle s\rangle}, \ldots, x_{i_{n}}^{\langle s\rangle}\right)$ describes one of the versions $A_{i}^{\langle s\rangle}$ of object $A_{i}$, and the component $x_{i_{i}}^{\langle s\rangle}=K_{l}\left(A_{i}^{\langle s\rangle}\right)$ is the attribute value $K_{l}$ in the $s$-th version $A_{i}^{\langle s\rangle}$ of object $A_{i}$, which is equal to $x^{j}, j=1, \ldots, h$ if all attributes $K_{1}, \ldots, K_{n}$ have the same scale of estimates $X=\left\{x^{1}, \ldots, x^{h}\right\}$, or $x_{i_{l}}^{e_{l}}$, $e_{l}=1, \ldots, h_{l}$ if attributes $K_{1}, \ldots, K_{n}$ have their own scales of estimates $X_{l}=\left\{x_{l}^{1}, \ldots, x_{l}^{h_{l}}\right\}, l=1, \ldots, n$.

The collection of objects $A_{1}, \ldots, A_{m}$, each of which exists in several versions $A_{i}^{\langle s\rangle}$, and their attributes can again be represented by the table Objects-Attributes $\mathrm{F}^{\#}=\left\|x_{i_{l}}^{\langle s\rangle}\right\|_{t m \times n}$, but have a larger dimension. The number of rows of matrix $\mathrm{F}^{\#}$ is equal to the number of all versions of objects, the number of columns is equal to the number of attributes, and elements $x_{i_{l}}^{\langle s\rangle}$ of the matrix are values of components $x_{i_{l}}^{\langle s\rangle}$ of vectors/tuples that specify different versions of objects.

Object $A_{i}$ is now represented in the $n$-dimensional space of attributes $X=X_{1} \times \ldots \times X_{n}$ not by a single point $\boldsymbol{x}_{i}$ but by an entire group (cloud) consisting of $t$ points $\left\{\boldsymbol{x}_{i}^{\langle 1\rangle}, \ldots, \boldsymbol{x}_{i}^{\langle t\rangle}\right\}$. It is important to note that the group of vectors/tuples $\boldsymbol{x}_{i}^{\langle 1\rangle}, \ldots, \boldsymbol{x}_{i}^{\langle i\rangle}$ that represent object $A_{i}$ should be treated as a single whole. In this case, generally speaking, individual values of attributes of different versions of object $A_{i}$ (estimates of different experts, characteristics measured in different ways or instruments) can be similar, different, and even contradictory, which in turn can lead to incomparability of vectors/tuples that represent the same object $A_{i}$.

The collection of such multi-attribute objects $A_{1}, \ldots$, $A_{m}$, each of which is represented in the attribute space $X=X_{1} \times \ldots \times X_{n}$ by its cloud consisting of $t$ different points, can have a rather complex structure that is difficult to analyze. Therefore, it is highly desirable to somehow simplify the description and aggregate the representation of such multi-attribute objects.

One possible approach to simplifying the description of the multi-attribute object $A_{i}$ represented by a group of vectors $\boldsymbol{x}_{i}^{\langle 1\rangle}, \ldots, \boldsymbol{x}_{i}^{\langle t\rangle}$ is as follows. Since components of any vector $x_{i_{l}}^{\langle s\rangle}=K_{l}\left(A_{i}^{\langle s\rangle}\right)$ are numerical values, in the attribute space $X=X_{1} \times \ldots \times X_{n}$, instead of a group of vectors, one can specify a single vector $\boldsymbol{x}_{i}^{\text {cond }}=$ $\left(x_{i_{1}}^{\text {cond }}, \ldots, x_{i_{n}}^{\text {cond }}\right)$, which will correspond to object $A_{i}$. The components of vector $\boldsymbol{x}_{i}^{\text {cond }}$ are determined from some additional significant considerations or formal conditions. As an example, it can be a vector that is the center of a group, a vector closest to all vectors in a group, or a vector with averaged or weighted values of components of vectors in a group.

However, when the attribute scales have discrete numerical estimates, the "averaged" vector may not physically exist in the original $n$-dimensional attribute space $X=X_{1} \times \ldots \times X_{n}$, since there are no corresponding numerical grades on the scales. In order to be able to operate with such vectors, it is necessary either to expand the initial scales of estimates by introducing intermediate numerical grades or to consider the scales of estimates to be continuous. Both, strictly speaking, change the original formulation of the problem. When objects are represented by tuples with symbolic, verbal or mixed values of components, a group of such tuples in principle cannot be replaced by a single tuple with averaged, weighted, or mixed estimates since such operations are mathematically not feasible.

If the group consists of only two vectors/tuples, then it is not possible to introduce some single object as a typical representative of the group, for example, one closest to all objects of the group. This is true for both numeric and symbolic attribute scales.

These and other similar difficulties can be overcome if we use the formalism of the theory of multisets, which allows us to take simultaneously heterogeneous attributes, possible combinations of attribute values, and the presence of different versions of objects into account.

Let all attributes $K_{1}, \ldots, K_{n}$ have the same scale of estimates $X=\left\{x^{1}, \ldots, x^{h}\right\}$. Let us choose set $X$ as a generating set and associate each object $A_{i}, i=1, \ldots, m$ with a multiset of the form (1)

$$
\boldsymbol{A}_{i}=\left\{k_{A_{i}}\left(x^{1}\right) \circ x^{1}, \ldots, k_{A_{i}}\left(x^{h}\right) \circ x^{h}\right\}
$$

over set $X=\left\{x^{1}, \ldots, x^{h}\right\}$. Here, the value of the multiplicity function $k_{A_{i}}\left(x^{j}\right)$ shows how many times estimate $x^{j}, j=1, \ldots, h$ is present in the description of object $A_{i}$. In this case, the collection of objects $A_{1}, \ldots$, $A_{m}$ and their attributes can be represented by the table 
Objects-Attributes $\mathrm{G}=\left\|k_{i j}\right\|_{m \times h}$. The rows of matrix $\mathrm{G}$ correspond to objects, the columns correspond to values of the $X$ scale of attributes, and the elements $k_{i j}$ correspond to the multiplicity values $k_{A_{i}}\left(x^{j}\right)$ of $x^{j}$ elements of the multisets defining the objects.

Let us suppose now that each attribute $K_{l}$ has its own scale of estimates $X_{l}=\left\{x_{l}^{1}, \ldots, x_{l}^{h_{l}}\right\}, l=1, \ldots, n$. Let us introduce a single scale (hyperscale) of attributes: set $X=X_{1} \bigcup \ldots \bigcup X_{n}=\left\{x_{1}^{1}, \ldots, x_{1}^{h_{1}} ; \ldots ; x_{n}^{1}, \ldots, x_{n}^{h_{n}}\right\}$, which consists of $n$ groups of attributes and combines all gradations of the estimates on the scales of all attributes. Then, each object $A_{i}$ will be associated with the multiset

$$
\begin{gathered}
\boldsymbol{A}_{i}=\left\{k_{\boldsymbol{A}_{i}}\left(x_{1}^{1}\right) \circ x_{1}^{1}, \ldots, k_{\boldsymbol{A}_{i}}\left(x_{1}^{h_{1}}\right) \circ x_{1}^{h_{1}} ; \ldots ;\right. \\
\left.k_{A_{i}}\left(x_{n}^{1}\right) \circ x_{n}^{1}, \ldots, k_{A_{i}}\left(x_{n}^{h_{n}}\right) \circ x_{n}^{h_{n}}\right\}
\end{gathered}
$$

over set $X=\left\{x_{1}^{1}, \ldots, x_{1}^{h_{1}} ; \ldots ; x_{n}^{1}, \ldots, x_{n}^{h_{n}}\right\}$ of gradations of scales of criteria. Here, the value of the multiplicity function $k_{A_{i}}\left(x_{l}^{e_{l}}\right)$ shows how many times estimate $x_{l}^{e_{l}} \in X_{l}, e_{l}=1, \ldots, h_{l}$ for attribute $K_{l}$ is present in the description of object $A_{i}$.

Despite the seemingly cumbersome representation of multi-attribute objects using multisets, these forms of notation are extremely convenient when comparing objects and performing operations, since calculations are carried out in parallel and simultaneously on all elements of all multisets. Let us note that expression (22) can also be easily written in the "usual" form (21) if we make a change of variables in set $X=\left\{x_{1}^{1}, \ldots, x_{1}^{h_{1}}\right.$; $\left.\ldots ; x_{n}^{1}, \ldots, x_{n}^{h_{n}}\right\}: x_{1}^{1}=x^{1}, \ldots, x_{1}^{h_{1}}=x^{h_{1}}, x_{2}^{1}=x^{h_{1}+1}, \ldots, x_{2}^{h_{2}}=$ $x^{h_{1}+h_{2}}, \ldots, x_{n}^{h_{n}}=x^{h}, h=h_{1}+\ldots+h_{n}$.

The collection of objects $A_{1}, \ldots, A_{m}$ and values of their attributes, represented by multisets of the form (22), can be defined by another table, Object-Attributes $\mathrm{H}=\left\|k_{i j}\right\|_{m \times h}, h=h_{1}+\ldots+h_{n}$. The rows of matrix $\mathrm{H}$ correspond to objects, the columns correspond to values of the attribute scales $X_{l}$, and the elements $k_{i j}$ correspond to the multiplicity values $k_{A_{i}}\left(x_{l}^{e_{l}}\right)$ of elements $x_{l}^{e_{l}}$ of the multisets defining the objects.

The variety of operations over multisets provides the ability to group multi-attribute objects in different ways. It is common to combine objects into groups using the operation of adding vectors or combining sets. In the case of multisets, group $D_{f}$ of objects can be formed as a sum $\boldsymbol{C}_{f}=\sum_{i} \boldsymbol{A}_{i}, k_{\boldsymbol{C}_{f}}\left(x^{j}\right)=\sum_{i} k_{\boldsymbol{A}_{i}}\left(x^{j}\right)$, union $\boldsymbol{C}_{f}=\bigcup_{i} \boldsymbol{A}_{i}, k_{\boldsymbol{C}_{f}}\left(x^{j}\right)=\max _{i} k_{\boldsymbol{A}_{i}}\left(x^{j}\right)$, intersection $\boldsymbol{C}_{f}=\bigcap_{i} \boldsymbol{A}_{i}, k_{C_{f}}\left(x^{j}\right)=\min _{i} k_{A_{i}}\left(x^{j}\right)$, or as a linear combination $\boldsymbol{C}_{f}=\sum_{i} b_{i} \cdot \boldsymbol{A}_{i}, \boldsymbol{C}_{f}=\bigcup_{i} b_{i} \cdot \boldsymbol{A}_{i}, \boldsymbol{C}_{f}=\bigcap_{i} b_{i} \cdot \boldsymbol{A}_{i}$ of multisets that define the objects. When group $D_{f}$ is formed by adding multisets, all properties (all values of all attributes) of all terms of the group are aggregated. When multisets are united or intersected, the best properties (maximum values of all attributes) or, respectively, the worst properties (minimum values of all attributes) for individual terms of the group increase.

Let us associate each version $A_{i}^{\langle s\rangle}, i=1, \ldots, m, s=$ $1, \ldots, t$ of the multi-attribute object $A_{i}$ with multiset $\boldsymbol{A}_{i}^{\langle s\rangle}=$ $\left\{k_{A_{i}}^{\langle s\rangle}\left(x^{1}\right) \circ x^{1}, \ldots ., k_{A_{i}}^{\langle s\rangle}\left(x^{h}\right) \circ x^{h}\right\}$, and each object $A_{i}$ with multiset $\boldsymbol{A}_{i}=\left\{k_{A_{i}}\left(x^{1}\right) \circ x^{1}, \ldots, k_{A_{i}}\left(x^{h}\right) \circ x^{h}\right\}$ over the set of estimates $X=\left\{x^{1}, \ldots, x^{h}\right\}$ or $X=X_{1} \bigcup \ldots \bigcup X_{n}=\left\{x_{1}^{1}, \ldots\right.$, $\left.x_{1}^{h_{1}} ; \ldots ; x_{n}^{1}, \ldots, x_{n}^{h_{n}}\right\}$. Multiset $\boldsymbol{A}_{i}$ representing object $\boldsymbol{A}_{i}$ as a whole will be formed by weighted addition of the multisets that describe the versions of this object: $\boldsymbol{A}_{i}=$ $c^{\langle 1\rangle} \boldsymbol{A}_{i}^{\langle 1\rangle}+\ldots+c^{\langle t\rangle} \boldsymbol{A}_{i}^{\langle t\rangle}$, where the multiplicity function is calculated by the rule $k_{A_{i}}\left(x^{j}\right)=\sum_{s} c^{\langle s\rangle} k_{A_{i}}^{\langle s\rangle}\left(x^{j}\right), j=1, \ldots$, h. $c^{\langle s\rangle}$ characterizes the significance of the $s$-th version (expert competence or measurement accuracy) whose values are sometimes normalized by $\sum_{s} c^{\langle s\rangle}=1$.

The objects $A_{1}, \ldots, A_{m}$, each of which exists in several versions, and the values of whose attributes are represented by multisets of the form (21) or (22), can be specified by the tables Objects-Attributes $\mathrm{G}^{\#}=$ $\left\|k_{i j}\right\|_{t m \times h}, \mathrm{H}^{\#}=\left\|k_{i j}\right\|_{t m \times h}$, or $\mathrm{H}=\left\|k_{i j}\right\|_{m \times h}, h=h_{1}+\ldots+h_{n}$. Elements $k_{i j}$ of matrices $\mathrm{G}^{\#}, \mathrm{H}^{\#}$, or $\mathrm{H}$ are multiplicities $k_{A_{i}}^{\langle s\rangle}\left(x^{j}\right), k_{A_{i}}^{\langle s\rangle}\left(x_{l}^{e_{l}}\right)$, or $k_{A_{i}}\left(x_{l}^{e_{l}}\right)$ of elements of the multisets that describe the corresponding versions $A_{i}^{\langle s\rangle}$ of objects $A_{i}$ or the objects $A_{i}$ themselves.

\section{AN ILLUSTRATIVE EXAMPLE}

There is a collection consisting of ten objects $A_{1}, \ldots$, $A_{10}$ described by eight attributes $K_{1}, \ldots, K_{8}$, each of which takes one of the values on a five-point scale of estimates $X=\left\{x^{1}, x^{2}, x^{3}, x^{4}, x^{5}\right\}$ [15]. As an example, objects $A_{1}, \ldots, A_{10}$ are survey questions for studying public opinion on a certain issue. Then, the attributes of the objects are the answers of eight respondents $K_{1}, \ldots$, $K_{8}$, which are coded as follows: $x^{1}, 1 /$ totally disagree; $x^{2}, 2 /$ disagree; $x^{3}, 3 /$ neutral; $x^{4}, 4 /$ agree; $x^{5}, 5 /$ completely agree. Alternately, the objects $A_{1}, \ldots, A_{10}$ could be schoolchildren and the object attributes are their annual scores in eight subjects: $K_{1}$, Mathematics; $K_{2}$, Physics; $K_{3}$, Chemistry; $K_{4}$, Biology; $K_{5}$, Geography; $K_{6}$, History; $K_{7}$, Literature; $K_{8}$, Foreign Language. The gradations of the estimates mean the following: $x^{1}$, $1 /$ very bad; $x^{2}, 2 /$ bad; $x^{3}, 3 /$ satisfactory; $x^{4}, 4 / \operatorname{good} ; x^{5}$, 5/excellent. 
Table 1, Objects-Attributes F, borrowed from [5] characterizes answers of university students who evaluated a course of lectures using numerical estimates. The same results of a student survey or schoolchildren scores recorded as multisets of numerical or verbal estimates are presented in Table 2, Objects-Attributes G. As an example, in Table 1 the answers of eight students to question $A_{1}$ are given by the vector/tuple of estimates $x_{1}=(4,5,4,5,4,5,4,5)$, while in Table 2 they are given by a multiset of estimates of the form (21): $\boldsymbol{A}_{1}=\left\{0 \circ x^{1}, 0 \circ x^{2}, 0 \circ x^{3}, 4 \circ x^{4}, 4 \circ x^{5}\right\}$. The last form of notation shows that object $A_{1}$ has four estimates $x^{4}$ that mean "agree" or "good," four estimates $x^{5}$ that mean "completely agree" or "excellent," and there are no other estimates for object $A_{1}$. If object $A_{1}$ is characterized by estimates for each of attributes $K_{1}$, ..., $K_{8}$ with different scales, then $A_{1}$ is represented by a multiset of the form (22):

$$
\begin{aligned}
\boldsymbol{A}_{1} & =\left\{0 \circ x_{1}^{1}, 0 \circ x_{1}^{2}, 0 \circ x_{1}^{3}, 1 \circ x_{1}^{4}, 0 \circ x_{1}^{5} ;\right. \\
& 0 \circ x_{2}^{1}, 0 \circ x_{2}^{2}, 0 \circ x_{2}^{3}, 0 \circ x_{2}^{4}, 1 \circ x_{2}^{5} ; \\
& 0 \circ x_{3}^{1}, 0 \circ x_{3}^{2}, 0 \circ x_{3}^{3}, 1 \circ x_{3}^{4}, 0 \circ x_{3}^{5} ; \\
& 0 \circ x_{4}^{1}, 0 \circ x_{4}^{2}, 0 \circ x_{4}^{3}, 0 \circ x_{4}^{4}, 1 \circ x_{4}^{5} ; \\
& 0 \circ x_{5}^{1}, 0 \circ x_{5}^{2}, 0 \circ x_{5}^{3}, 1 \circ x_{5}^{4}, 0 \circ x_{5}^{5} ; \\
& 0 \circ x_{6}^{1}, 0 \circ x_{6}^{2}, 0 \circ x_{6}^{3}, 0 \circ x_{6}^{4}, 1 \circ x_{6}^{5} ; \\
& 0 \circ x_{7}^{1}, 0 \circ x_{7}^{2}, 0 \circ x_{7}^{3}, 1 \circ x_{7}^{4}, 0 \circ x_{7}^{5} ; \\
& \left.0 \circ x_{8}^{1}, 0 \circ x_{8}^{2}, 0 \circ x_{8}^{3}, 0 \circ x_{8}^{4}, 1 \circ x_{8}^{5}\right\} .
\end{aligned}
$$

Now, let each object be present in several instances (versions or copies) differing between each other. As an example, eight respondents $K_{1}, \ldots, K_{8}$, who gave answers to the same questions $A_{1}, \ldots, A_{10}$, were polled twice. Alternatively, schoolchildren $A_{1}, \ldots, A_{10}$ received scores for the same eight disciplines $K_{1}, \ldots, K_{8}$ in each semester, that is, twice a year. Thus, each object will be specified not by one but by two vectors/tuples of attributes, or by two multisets. Formally, we can assume that the method used to describe versions of the object is an expression of the individual opinion of an expert, and the description of the object "in general" is an aggregate collective judgment of two experts.

The different versions of the objects are defined in Tables 3 and 4, Objects-Attributes $\mathrm{F}^{\#}$ and $\mathrm{G}^{\#}$, respectively. In Table 3 , object $A_{1}$ is represented by a group of two vectors/tuples $\boldsymbol{x}_{1}^{\langle 1\rangle}=(4,5,4,5,4,5,4,5)$ and $\boldsymbol{x}_{1}^{\langle 2\rangle}=$ $(5,5,5,5,4,4,4,5)$. The aggregated estimates of object $A_{1}$, when averaged, will be described by vector $\boldsymbol{x}_{1}^{\mathrm{av}}=(4.5,5.0,4.5,5.0,4.0,4.5,4.0,5.0)$ of the "average" estimates, although non-integer numbers are absent in the established five-point scale $X=\{1,2,3$, $4,5\}$. Two versions of object $A_{1}$ are represented in Table 4 by multisets of estimates of the form (21) $\boldsymbol{A}_{1}^{\langle 1\rangle}=$
Table 1. Data table $\mathrm{F}$

\begin{tabular}{l|c|c|c|c|c|c|c|c}
\hline$A \backslash K$ & $K_{1}$ & $K_{2}$ & $K_{3}$ & $K_{4}$ & $K_{5}$ & $K_{6}$ & $K_{7}$ & $K_{8}$ \\
\hline$A_{1}$ & 4 & 5 & 4 & 5 & 4 & 5 & 4 & 5 \\
$A_{2}$ & 4 & 1 & 2 & 1 & 3 & 2 & 2 & 2 \\
$A_{3}$ & 1 & 1 & 3 & 1 & 4 & 1 & 1 & 4 \\
$A_{4}$ & 5 & 3 & 2 & 4 & 4 & 5 & 4 & 5 \\
$A_{5}$ & 4 & 4 & 4 & 4 & 4 & 5 & 4 & 4 \\
$A_{6}$ & 5 & 5 & 4 & 4 & 4 & 5 & 5 & 4 \\
$A_{7}$ & 4 & 1 & 2 & 3 & 3 & 3 & 1 & 2 \\
$A_{8}$ & 4 & 5 & 4 & 2 & 3 & 4 & 5 & 3 \\
$A_{9}$ & 3 & 2 & 3 & 1 & 3 & 3 & 2 & 2 \\
$A_{10}$ & 5 & 5 & 4 & 5 & 3 & 5 & 5 & 4
\end{tabular}

Table 2. Data table G

\begin{tabular}{l|c|c|c|c|c}
\hline \multicolumn{1}{c|}{$A \backslash X$} & $x^{1}$ & $x^{2}$ & $x^{3}$ & $x^{4}$ & $x^{5}$ \\
\hline$A_{1}$ & 0 & 0 & 0 & 4 & 4 \\
$A_{2}$ & 2 & 4 & 1 & 1 & 0 \\
$A_{3}$ & 5 & 0 & 1 & 2 & 0 \\
$A_{4}$ & 0 & 1 & 1 & 3 & 3 \\
$A_{5}$ & 0 & 0 & 0 & 7 & 1 \\
$A_{6}$ & 0 & 0 & 0 & 4 & 4 \\
$A_{7}$ & 2 & 2 & 3 & 1 & 0 \\
$A_{8}$ & 0 & 1 & 2 & 3 & 2 \\
$A_{9}$ & 1 & 3 & 4 & 0 & 0 \\
$A_{10}$ & 0 & 0 & 1 & 2 & 5 \\
\hline
\end{tabular}

$\left\{0 \circ x^{1}, 0 \circ x^{2}, 0 \circ x^{3}, 4 \circ x^{4}, 4 \circ x^{5}\right\}$ and $A_{1}^{\langle 2\rangle}=\left\{0 \circ x^{1}, 0 \circ x^{2}\right.$, $\left.0 \circ x^{3}, 3 \circ x^{4}, 5 \circ x^{5}\right\}$. For simplicity, we will consider the respondents as equally competent or semester scores to be equally significant: $c^{\langle 1\rangle}=c^{\langle 2\rangle}=1$. Then, object $A_{1}$ "as a whole" is described by multiset

$$
\boldsymbol{A}_{1}=\boldsymbol{A}_{1}^{\langle 1\rangle}+\boldsymbol{A}_{1}^{\langle 2\rangle}=\left\{0 \circ x^{1}, 0 \circ x^{2}, 0 \circ x^{3}, 7 \circ x^{4}, 9 \circ x^{5}\right\} .
$$

Similarly, objects $A_{2}, A_{4}, A_{6}, A_{7}$, and $A_{8}$ are defined by multisets

$$
\begin{aligned}
& A_{2}=A_{2}^{\langle 1\rangle}+A_{2}^{\langle 2\rangle}=\left\{4 \circ x^{1}, 6 \circ x^{2}, 4 \circ x^{3}, 2 \circ x^{4}, 0 \circ x^{5}\right\}, \\
& A_{4}=A_{4}^{\langle 1\rangle}+A_{4}^{\langle 2\rangle}=\left\{0 \circ x^{1}, 1 \circ x^{2}, 3 \circ x^{3}, 7 \circ x^{4}, 5 \circ x^{5}\right\}, \\
& A_{6}=A_{6}^{\langle 1\rangle}+A_{6}^{\langle 2\rangle}=\left\{0 \circ x^{1}, 0 \circ x^{2}, 0 \circ x^{3}, 9 \circ x^{4}, 7 \circ x^{5}\right\}, \\
& A_{7}=A_{7}^{\langle 1\rangle}+A_{7}^{\langle 2\rangle}=\left\{3 \circ x^{1}, 5 \circ x^{2}, 5 \circ x^{3}, 3 \circ x^{4}, 0 \circ x^{5}\right\}, \\
& A_{8}=A_{8}^{\langle 1\rangle}+A_{8}^{\langle 2\rangle}=\left\{0 \circ x^{1}, 1 \circ x^{2}, 3 \circ x^{3}, 7 \circ x^{4}, 5 \circ x^{5}\right\} .
\end{aligned}
$$


Table 3. Data table $\mathrm{F}^{\#}$

\begin{tabular}{l|c|c|c|c|c|c|c|c}
\hline$A \backslash K$ & $K_{1}$ & $K_{2}$ & $K_{3}$ & $K_{4}$ & $K_{5}$ & $K_{6}$ & $K_{7}$ & $K_{8}$ \\
\hline$A_{1}$ & 4 & 5 & 4 & 5 & 4 & 5 & 4 & 5 \\
$A_{2}$ & 5 & 5 & 5 & 5 & 4 & 4 & 4 & 5 \\
& 4 & 1 & 2 & 1 & 3 & 2 & 2 & 2 \\
$A_{3}$ & 3 & 2 & 1 & 1 & 4 & 3 & 3 & 2 \\
& 1 & 1 & 3 & 1 & 4 & 1 & 1 & 4 \\
$A_{4}$ & 5 & 2 & 3 & 1 & 5 & 2 & 1 & 3 \\
& 4 & 4 & 3 & 5 & 4 & 5 & 3 & 4 \\
$A_{5}$ & 4 & 4 & 4 & 4 & 4 & 5 & 4 & 4 \\
& 5 & 5 & 3 & 4 & 4 & 4 & 5 & 4 \\
$A_{6}$ & 5 & 5 & 4 & 4 & 4 & 5 & 5 & 4 \\
& 4 & 5 & 4 & 4 & 4 & 4 & 5 & 5 \\
$A_{7}$ & 4 & 1 & 2 & 3 & 3 & 3 & 1 & 2 \\
& 3 & 2 & 1 & 4 & 2 & 4 & 2 & 3 \\
$A_{8}$ & 4 & 5 & 4 & 2 & 3 & 4 & 5 & 3 \\
& 5 & 4 & 5 & 3 & 4 & 5 & 4 & 4 \\
$A_{9}$ & 3 & 2 & 3 & 1 & 3 & 3 & 2 & 2 \\
& 4 & 3 & 2 & 2 & 2 & 3 & 3 & 2 \\
$A_{10}$ & 5 & 5 & 4 & 5 & 3 & 5 & 5 & 4 \\
& 3 & 4 & 3 & 4 & 2 & 4 & 2 & 4 \\
\hline \multirow{4}{*}{} & & & & & & & & 5
\end{tabular}

Table 4. Data table $\mathrm{G}^{\#}$

\begin{tabular}{|c|c|c|c|c|c|}
\hline$A \backslash X$ & $x^{1}$ & $x^{2}$ & $x^{3}$ & $x^{4}$ & $x^{5}$ \\
\hline \multirow[t]{2}{*}{$A_{1}$} & 0 & 0 & 0 & 4 & 4 \\
\hline & 0 & 0 & 0 & 3 & 5 \\
\hline \multirow[t]{2}{*}{$A_{2}$} & 2 & 4 & 1 & 1 & 0 \\
\hline & 2 & 2 & 3 & 1 & 0 \\
\hline \multirow[t]{2}{*}{$A_{3}$} & 5 & 0 & 1 & 2 & 0 \\
\hline & 3 & 2 & 2 & 0 & 1 \\
\hline \multirow[t]{2}{*}{$A_{4}$} & 0 & 1 & 1 & 3 & 3 \\
\hline & 0 & 0 & 2 & 4 & 2 \\
\hline \multirow[t]{2}{*}{$A_{5}$} & 0 & 0 & 0 & 7 & 1 \\
\hline & 0 & 0 & 1 & 4 & 3 \\
\hline \multirow[t]{2}{*}{$A_{6}$} & 0 & 0 & 0 & 4 & 4 \\
\hline & 0 & 0 & 0 & 5 & 3 \\
\hline \multirow[t]{2}{*}{$A_{7}$} & 2 & 2 & 3 & 1 & 0 \\
\hline & 1 & 3 & 2 & 2 & 0 \\
\hline \multirow[t]{2}{*}{$A_{8}$} & 0 & 1 & 2 & 3 & 2 \\
\hline & 0 & 0 & 1 & 4 & 3 \\
\hline \multirow[t]{2}{*}{$A_{9}$} & 1 & 3 & 4 & 0 & 0 \\
\hline & 0 & 4 & 3 & 1 & 0 \\
\hline \multirow[t]{2}{*}{$A_{10}$} & 0 & 0 & 1 & 2 & 5 \\
\hline & 0 & 2 & 2 & 4 & 0 \\
\hline
\end{tabular}

Table 5 Objects-Attributes $\mathrm{H}$ shows objects $A_{1}, \ldots$, $A_{10}$ "generally" and the values of their attributes given by multisets of the form (22). Thus, for example, object $A_{1}$ is associated with the following multiset of estimates:

$$
\begin{aligned}
\boldsymbol{A}_{1} & =\left\{0 \circ x_{1}^{1}, 0 \circ x_{1}^{2}, 0 \circ x_{1}^{3}, 1 \circ x_{1}^{4}, 1 \circ x_{1}^{5} ;\right. \\
& 0 \circ x_{2}^{1}, 0 \circ x_{2}^{2}, 0 \circ x_{2}^{3}, 0 \circ x_{2}^{4}, 2 \circ x_{2}^{5} \\
& 0 \circ x_{3}^{1}, 0 \circ x_{3}^{2}, 0 \circ x_{3}^{3}, 1 \circ x_{3}^{4}, 1 \circ x_{3}^{5} ; \\
& 0 \circ x_{4}^{1}, 0 \circ x_{4}^{2}, 0 \circ x_{4}^{3}, 0 \circ x_{4}^{4}, 2 \circ x_{4}^{5} \\
& 0 \circ x_{5}^{1}, 0 \circ x_{5}^{2}, 0 \circ x_{5}^{3}, 2 \circ x_{5}^{4}, 0 \circ x_{5}^{5} \\
& 0 \circ x_{6}^{1}, 0 \circ x_{6}^{2}, 0 \circ x_{6}^{3}, 1 \circ x_{6}^{4}, 1 \circ x_{6}^{5} ; \\
& 0 \circ x_{7}^{1}, 0 \circ x_{7}^{2}, 0 \circ x_{7}^{3}, 2 \circ x_{7}^{4}, 0 \circ x_{7}^{5} \\
& \left.0 \circ x_{8}^{1}, 0 \circ x_{8}^{2}, 0 \circ x_{8}^{3}, 0 \circ x_{8}^{4}, 2 \circ x_{8}^{5}\right\}
\end{aligned}
$$

The table Objects-Attributes $\mathrm{H}^{\#}$, which represents individual versions of objects and their attributes, is rather cumbersome and is not given for brevity.

The proximity of objects in the space of attributes is characterized either by their difference or by the similarity of their properties. We will consider the multiattribute object $A_{i}$ represented by multiset $\boldsymbol{A}_{i}$ as a point of the metric space of multisets $(\boldsymbol{A}, d), \boldsymbol{A}=\left\{\boldsymbol{A}_{1}, \ldots, \boldsymbol{A}_{m}\right\}$, where $d$ is one of the metrics (9)-(20). In practical problems, the difference between objects $\boldsymbol{A}_{i}$ and $\boldsymbol{A}_{j}$ is conveniently estimated by one of the metrics (17)(20), which we write in the following form:

$$
\begin{gathered}
d_{Z_{1 p}}\left(\boldsymbol{A}_{i}, \boldsymbol{A}_{j}\right)=\left[S_{i j}\right]^{1 / p} ; \\
d_{Z_{2 p}}\left(\boldsymbol{A}_{i}, \boldsymbol{A}_{j}\right)=\left[S_{i j} / W\right]^{1 / p} ; \\
d_{Z_{3 p}}\left(\boldsymbol{A}_{i}, \boldsymbol{A}_{j}\right)=\left[S_{i j} / M_{i j}\right]^{1 / p} ; \\
d_{Z_{4 p}}\left(\boldsymbol{A}_{i}, \boldsymbol{A}_{j}\right)=\left[S_{i j} / N_{i j}\right]^{1 / p} .
\end{gathered}
$$

Here,

$$
\begin{aligned}
S_{i j}= & m(\boldsymbol{A} \Delta \boldsymbol{B})=\sum_{l=1}^{n} w_{l}\left|k_{A_{i}}\left(x^{l}\right)-k_{A_{j}}\left(x^{l}\right)\right| \\
= & \sum_{l=1}^{n} w_{l} \sum_{e_{l}=1}^{h_{l}} \mid k_{A_{i}}\left(x_{l}^{e_{l}}\right)-k_{A_{j}}\left(x_{l}^{e_{l}}\right) ; \\
L_{i j}= & m(\boldsymbol{A} \bigcap \boldsymbol{B})=\sum_{l=1}^{n} w_{l} \min \left|k_{\boldsymbol{A}_{i}}\left(x^{l}\right), k_{\boldsymbol{A}_{j}}\left(x^{l}\right)\right| \\
= & \sum_{l=1}^{n} w_{l} \sum_{e_{l}=1}^{h_{l}} \min \left|k_{\boldsymbol{A}_{i}}\left(x_{l}^{e_{l}}\right), k_{\boldsymbol{A}_{j}}\left(x_{l}^{e_{l}}\right)\right| ; \\
M_{i j}= & m(\boldsymbol{A} \bigcup \boldsymbol{B})=\sum_{l=1}^{n} w_{l} \max \left|k_{\boldsymbol{A}_{i}}\left(x^{l}\right), k_{\boldsymbol{A}_{j}}\left(x^{l}\right)\right| \\
= & \sum_{l=1}^{n} w_{l} \sum_{e_{l}=1}^{h_{l}} \max \left|k_{\boldsymbol{A}_{i}}\left(x_{l}^{e_{l}}\right), k_{\boldsymbol{A}_{j}}\left(x_{l}^{e_{l}}\right)\right| ;
\end{aligned}
$$


Table 5. Data table $\mathrm{H}$

\begin{tabular}{l|c|c|c|c|c|c|c|c|c}
\hline \multicolumn{1}{c|}{$A \backslash X$} & $x_{1}^{1} x_{1}^{2} x_{1}^{3} x_{1}^{4} x_{1}^{5}$ & $x_{2}^{1} x_{2}^{2} x_{2}^{3} x_{2}^{4} x_{2}^{5}$ & $x_{1}^{1} x_{3}^{2} x_{3}^{3} x_{3}^{4} x_{3}^{5}$ & $x_{4}^{1} x_{4}^{2} x_{4}^{3} x_{4}^{4} x_{4}^{5}$ & $x_{5}^{1} x_{5}^{2} x_{5}^{3} x_{5}^{4} x_{5}^{5}$ & $x_{6}^{1} x_{6}^{2} x_{6}^{3} x_{6}^{4} x_{6}^{5}$ & $x_{7}^{1} x_{7}^{2} x_{7}^{3} x_{7}^{4} x_{7}^{5}$ & $x_{8}^{1} x_{8}^{2} x_{8}^{3} x_{8}^{4} x_{8}^{5}$ \\
\hline$A_{1}$ & 000011 & 00000 & 000011 & 00002 & 00020 & 00011 & 00020 & 00002 \\
$A_{2}$ & 00110 & 11000 & 11000 & 20000 & 00110 & 01100 & 01100 & 02000 \\
$A_{3}$ & 200000 & 11000 & 00200 & 20000 & 00011 & 11000 & 20000 & 00110 \\
$A_{4}$ & 000011 & 00110 & 01100 & 00011 & 00020 & 00002 & 00110 & 00011 \\
$A_{5}$ & 00011 & 00011 & 00110 & 00020 & 00020 & 00011 & 00011 & 00020 \\
$A_{6}$ & 00011 & 00002 & 00020 & 00020 & 00020 & 00011 & 00002 & 00011 \\
$A_{7}$ & 00110 & 11000 & 11000 & 00110 & 01100 & 00110 & 11000 & 01100 \\
$A_{8}$ & 00011 & 00011 & 00011 & 01100 & 00110 & 00011 & 00011 & 00110 \\
$A_{9}$ & 00110 & 01100 & 01100 & 11000 & 01100 & 00200 & 01100 & 00200 \\
$A_{10}$ & 00101 & 00011 & 00110 & 00011 & 01100 & 00011 & 01001 & 00020 \\
\hline
\end{tabular}

$$
\begin{gathered}
N_{i j}=m(\boldsymbol{A}+\boldsymbol{B})=\sum_{l=1}^{n} w_{l}\left|k_{\boldsymbol{A}_{i}}\left(x^{l}\right)+k_{\boldsymbol{A}_{j}}\left(x^{l}\right)\right| \\
=\sum_{l=1}^{n} w_{l} \sum_{e_{l}=1}^{h_{l}}\left|k_{\boldsymbol{A}_{i}}\left(x_{l}^{e_{l}}\right)+k_{\boldsymbol{A}_{j}}\left(x_{l}^{e_{l}}\right)\right| \\
W=m(\boldsymbol{Z})=\sum_{l=1}^{n} w_{l} \max _{\boldsymbol{A} \in \boldsymbol{A}} k_{\boldsymbol{A}}\left(x^{l}\right) \\
=\sum_{l=1}^{n} w_{l} \sum_{e_{l}=1}^{h_{l}} \max _{\boldsymbol{A} \in \boldsymbol{A}} k_{\boldsymbol{A}}\left(x_{l}^{e_{l}}\right) .
\end{gathered}
$$

The measure of the multiset is given as a linear combination (6) of multiplicity functions: $m(\boldsymbol{A})=$ $\sum_{l=1}^{n} w_{l} k_{A}\left(x^{l}\right)=\sum_{l=1}^{n} w_{l} \sum_{e_{l}=1}^{h_{l}} k_{A}\left(x_{l}^{e_{l}}\right)$, where $w_{i}>0$ is the significance (weight) of element $x_{i}$ or, which is the same, of attribute $K_{l}, l=1, \ldots, n$. For quantities $L_{i j}, M_{i j}$, $N_{i j}$, and $S_{i j}$, relations $N_{i j}=M_{i j}+L_{i j}, S_{i j}=M_{i j}-L_{i j}$ are satisfied.

The similarity between objects can be expressed by one of the following indicators:

$$
\begin{gathered}
s_{0}\left(\boldsymbol{A}_{i}, \boldsymbol{A}_{j}\right)=L_{i j} ; \quad s_{1}\left(\boldsymbol{A}_{i}, \boldsymbol{A}_{j}\right)=L_{i j} / W ; \\
s_{2}\left(\boldsymbol{A}_{i}, \boldsymbol{A}_{j}\right)=1-d_{21}\left(\boldsymbol{A}_{i}, \boldsymbol{A}_{j}\right)=1-\left(S_{i j} / W\right) ; \\
s_{3}\left(\boldsymbol{A}_{i}, \boldsymbol{A}_{j}\right)=1-d_{31}\left(\boldsymbol{A}_{i}, \boldsymbol{A}_{j}\right)=L_{i j} / M_{i j} ; \\
s_{4}\left(\boldsymbol{A}_{i}, \boldsymbol{A}_{j}\right)=1-d_{41}\left(\boldsymbol{A}_{i}, \boldsymbol{A}_{j}\right)=2 L_{i j} / N_{i j} .
\end{gathered}
$$

Expressions $s_{0}, s_{1}, s_{2}, s_{3}$, and $s_{4}(24)$ generalize for the case of multisets the known nonmetric indices of the similarity of objects such as, respectively, the measure of absolute similarity, the Russell-Rao similarity measure, the simple matching coefficient, the Jacquard coefficient or the Rogers-Tanimoto measure, and the Sørensen index [4, 18].

In the example, the closest in the space of multisets $\left(\boldsymbol{A}, d_{11}\right)$ are objects $A_{4}$ and $A_{8}$, which lie at a distance

$$
d_{11}\left(A_{4}, A_{8}\right)=|0-0|+|0-0|+|0-0|+|0-0|+|0-0|=0 \text {. }
$$

The group $D_{01}$, which includes objects $A_{4}$ and $A_{8}$, is associated with multiset $\boldsymbol{C}_{01}$ formed by adding the multisets:

$$
\begin{aligned}
\boldsymbol{C}_{01}= & A_{4}+\boldsymbol{A}_{8}=\left\{0 \circ x^{1}, 1 \circ x^{2}, 3 \circ x^{3}, 7 \circ x^{4}, 5 \circ x^{5}\right\} \\
& +\left\{0 \circ x^{1}, 1 \circ x^{2}, 3 \circ x^{3}, 7 \circ x^{4}, 5 \circ x^{5}\right\} \\
= & \left\{0 \circ x_{1}, 2 \circ x_{2}, 6 \circ x_{3}, 14 \circ x_{4}, 10 \circ x_{5}\right\} .
\end{aligned}
$$

Two pairs of objects $A_{1}$ and $A_{6}, A_{2}$ and $A_{7}$ are at the same distances:

$$
\begin{aligned}
& d_{11}\left(\boldsymbol{A}_{1}, \boldsymbol{A}_{6}\right)=|0-0|+|0-0|+|0-0|+|7-9|+|9-7|=4, \\
& d_{11}\left(\boldsymbol{A}_{2}, \boldsymbol{A}_{7}\right)=|4-3|+|6-5|+|4-5|+|2-3|+|0-0|=4 .
\end{aligned}
$$

Objects $A_{1}$ and $A_{6}$ form group $D_{02}$ represented by multiset

$$
\begin{aligned}
\boldsymbol{C}_{02}= & A_{1}+A_{6}=\left\{0 \circ x^{1}, 0 \circ x^{2}, 0 \circ x^{3}, 7 \circ x^{4}, 9 \circ x^{5}\right\} \\
& +\left\{0 \circ x^{1}, 0 \circ x^{2}, 0 \circ x^{3}, 9 \circ x^{4}, 7 \circ x^{5}\right\} \\
= & \left\{0 \circ x^{1}, 0 \circ x^{2}, 0 \circ x^{3}, 16 \circ x^{4}, 16 \circ x^{5}\right\},
\end{aligned}
$$

and objects $A_{2}$ and $A_{7}$ form group $D_{03}$ represented by multiset

$$
\begin{aligned}
\boldsymbol{C}_{03}= & \boldsymbol{A}_{12}+\boldsymbol{A}_{7}=\left\{4 \circ x^{1}, 6 \circ x^{2}, 4 \circ x^{3}, 2 \circ x^{4}, 0 \circ x^{5}\right\} \\
& +\left\{3 \circ x^{1}, 5 \circ x^{2}, 5 \circ x^{3}, 3 \circ x^{4}, 0 \circ x^{5}\right\} \\
& =\left\{7 \circ x^{1}, 11 \circ x^{2}, 9 \circ x^{3}, 5 \circ x^{4}, 0 \circ x^{5}\right\} .
\end{aligned}
$$

The suggested indices of dissimilarity (23) and similarity (24) between multi-attribute objects were used in the methods of ordering ARAMIS (Aggregation and Ranking of Alternatives nearby Multi-attribute Ideal Situations), cluster analysis CLAVA-HI (CLuster Analysis of Verbal Alternatives-HIerarchical) and CLAVA-NI (CLuster Analysis of Verbal AlternativesNon-Ierarchical), and classification MASKA (MultiAttribute Consistent Classification of Alternatives) for 
objects with mismatched attribute values [13, 15]. Examples of practical application of these methods in the problems of group multicriterion decision-making were given in [14-17].

\section{CONCLUSIONS}

This paper describes new classes of spaces of finite, bounded, and measurable sets and multisets and new types of metrics that can be used in solving various problems of classification, sorting, and ranking of multi-attribute objects. Representation of multi-attribute objects using multisets and application of new types of indicators of proximity of objects make it possible to expand the range of problems, as well as to more easily find solutions to known problems.

\section{APPENDIX}

Let us verify that the function $d_{Z_{4 p}}(\boldsymbol{A}, \boldsymbol{B})=$ $[m(\boldsymbol{A} \Delta \boldsymbol{B}) / m(\boldsymbol{A}+\boldsymbol{B})]^{1 / p}$ defined on $\sigma$-algebra $\boldsymbol{S}_{\sigma}(\boldsymbol{Z})$ of multisets is quasi-metric (symmetric almost everywhere) on space $\left(\boldsymbol{S}_{\sigma}(\boldsymbol{Z}), m\right)$ of multisets with a strongly $\sigma$-additive and completely $\sigma$-finite measure.

The validity and nonnegativity of function $d_{Z_{4 p}}(A, B)$ follow from the definition of measure $m$ as a real and nonnegative multiset function defined on $\sigma$ algebra $\boldsymbol{S}_{\sigma}(\boldsymbol{Z})$ of multisets. The axiom of symmetry $\left(1^{0}\right)$ follows from the commutativity of the addition operations $\boldsymbol{A}+\boldsymbol{B}=\boldsymbol{B}+\boldsymbol{A}$ and symmetric difference $\boldsymbol{A} \Delta \boldsymbol{B}=\boldsymbol{B} \Delta \boldsymbol{A}$ of multisets.

Let us show using examples that the triangle inequality $\left(3^{0}\right)$ can be both satisfied and not for function $d_{Z_{4 p}}(\boldsymbol{A}, \boldsymbol{B})$. For $\boldsymbol{C} \subseteq A \bigcap \boldsymbol{B}$, by the monotonicity property of a measure of a multiset, we have $m(\boldsymbol{C}) \leq$
$m(\boldsymbol{A} \bigcap \boldsymbol{B}) \leq m(\boldsymbol{A}), m(\boldsymbol{C}) \leq m(\boldsymbol{A} \bigcap \boldsymbol{B}) \leq m(\boldsymbol{B})$. Then, taking the inequality $m(\boldsymbol{A} \Delta \boldsymbol{B}) \leq m[(\boldsymbol{A} \Delta \boldsymbol{C})+(\boldsymbol{B} \Delta \boldsymbol{C})]=$ $m(\boldsymbol{A} \Delta \boldsymbol{C})+m(\boldsymbol{C} \Delta \boldsymbol{B})$ proved in [6,7] into account we obtain:

$$
\begin{gathered}
m(\boldsymbol{A} \Delta \boldsymbol{B}) / m(\boldsymbol{A}+\boldsymbol{B}) \\
\leq[m(\boldsymbol{A} \Delta \boldsymbol{C})+m(\boldsymbol{B} \Delta \boldsymbol{C})] /[m(\boldsymbol{A})+m(\boldsymbol{B})] \\
\leq m(\boldsymbol{A} \Delta \boldsymbol{C}) / m(\boldsymbol{A}+\boldsymbol{C})+m(\boldsymbol{B} \Delta \boldsymbol{C}) / m(\boldsymbol{B}+\boldsymbol{C}) .
\end{gathered}
$$

Therefore,

$$
\begin{gathered}
{\left[d_{\boldsymbol{Z}_{4 p}}(\boldsymbol{A}, \boldsymbol{B})\right]^{p}=m(\boldsymbol{A} \Delta \boldsymbol{B}) / m(\boldsymbol{A}+\boldsymbol{B})} \\
\leq m(\boldsymbol{A} \Delta \boldsymbol{C}) / m(\boldsymbol{A}+\boldsymbol{C})+m(\boldsymbol{B} \Delta \boldsymbol{C}) / m(\boldsymbol{B}+\boldsymbol{C}) \\
\leq\left[d_{\boldsymbol{Z}_{4 p}}(\boldsymbol{A}, \boldsymbol{C})\right]^{p}+\left[d_{\boldsymbol{Z}_{4 p}}(\boldsymbol{B}, \boldsymbol{C})\right]^{p} \\
\leq\left[d_{\boldsymbol{Z}_{4 p}}(\boldsymbol{A}, \boldsymbol{C})+d_{\boldsymbol{Z}_{4 p}}(\boldsymbol{B}, \boldsymbol{C})\right]^{p}
\end{gathered}
$$

and

$$
d_{Z_{4 p}}(\boldsymbol{A}, \boldsymbol{B}) \leq d_{Z_{4 p}}(\boldsymbol{A}, \boldsymbol{C})+d_{Z_{4 p}}(\boldsymbol{B}, \boldsymbol{C}) .
$$

On the other hand, for arbitrary multisets $\boldsymbol{A}, \boldsymbol{B}$, and $\boldsymbol{C}$, from formulas (7)-(8), we have

$$
\begin{gathered}
m(\boldsymbol{A} \bigcup \boldsymbol{B})=m(\boldsymbol{A})+m(\boldsymbol{B}-\boldsymbol{A})=m(\boldsymbol{B})+m(\boldsymbol{A}-\boldsymbol{B}), \\
m(\boldsymbol{A} \Delta \boldsymbol{B})=m(\boldsymbol{A}-\boldsymbol{B})+m(\boldsymbol{B}-\boldsymbol{A}), \\
m(\boldsymbol{A} \Delta(\boldsymbol{A} \bigcup \boldsymbol{B}))=m(\boldsymbol{A} \bigcup \boldsymbol{B})-m(\boldsymbol{A})=m(\boldsymbol{B}-\boldsymbol{A}), \\
m(\boldsymbol{B} \Delta(\boldsymbol{A} \bigcup \boldsymbol{B}))=m(\boldsymbol{A} \bigcup \boldsymbol{B})-m(\boldsymbol{B}) \\
=m(\boldsymbol{A}-\boldsymbol{B}) .
\end{gathered}
$$

Then for $\boldsymbol{C}=\boldsymbol{A} \bigcup \boldsymbol{B}$, taking into account the inequality $m(\boldsymbol{A} \bigcap \boldsymbol{B}) \leq m(\boldsymbol{A}), m(\boldsymbol{B}) \leq m(\boldsymbol{A} \bigcup \boldsymbol{B}) \leq$ $m(\boldsymbol{A}+\boldsymbol{B})$, which follows from (8), we obtain

$$
\begin{gathered}
{\left[d_{Z_{4 p}}(\boldsymbol{A}, \boldsymbol{B})\right]^{p}=m(\boldsymbol{A} \Delta \boldsymbol{B}) / m(\boldsymbol{A}+\boldsymbol{B})} \\
=m(\boldsymbol{B}-\boldsymbol{A}) /[m(\boldsymbol{A})+m(\boldsymbol{B})]+m(\boldsymbol{A}-\boldsymbol{B}) /[m(\boldsymbol{B})+m(\boldsymbol{A})] \\
\geq m(\boldsymbol{B}-\boldsymbol{A}) /[m(\boldsymbol{A})+m(\boldsymbol{B})+m(\boldsymbol{A}-\boldsymbol{B})]+m(\boldsymbol{A}-\boldsymbol{B}) /[m(\boldsymbol{B})+m(\boldsymbol{A})+m(\boldsymbol{B}-\boldsymbol{A})] \\
=m(\boldsymbol{A} \Delta(\boldsymbol{A} \bigcup \boldsymbol{B})) /[m(\boldsymbol{A})+m(\boldsymbol{A} \bigcup \boldsymbol{B})]+m(\boldsymbol{B} \Delta(\boldsymbol{A} \bigcup \boldsymbol{B})) /[m(\boldsymbol{B})+m(\boldsymbol{A} \bigcup \boldsymbol{B})] \\
=m(\boldsymbol{A} \Delta \boldsymbol{C}) / m(\boldsymbol{A}+\boldsymbol{C})+m(\boldsymbol{B} \Delta \boldsymbol{C}) / m(\boldsymbol{B}+\boldsymbol{C})=\left[d_{Z_{4 p}}(\boldsymbol{A}, \boldsymbol{C})\right]^{p}+\left[d_{Z_{4 p}}(\boldsymbol{B}, \boldsymbol{C})\right]^{p} .
\end{gathered}
$$

It can be that $d_{Z_{4 p}}(\boldsymbol{A}, \boldsymbol{B}) \geq d_{Z_{4 p}}(\boldsymbol{A}, \boldsymbol{C})+d_{Z_{4 p}}(\boldsymbol{B}, \boldsymbol{C})$.

By the identity property of the symmetric difference of multisets $\boldsymbol{A} \Delta \boldsymbol{B}=\varnothing$ for $\boldsymbol{A}=\boldsymbol{B}$. Then, $m(\boldsymbol{A} \Delta \boldsymbol{B})=$ $m(\varnothing)=0$ and $d_{Z_{4 p}}(\boldsymbol{A}, \boldsymbol{B})=0$. At the same time, the condition $m(\boldsymbol{A} \Delta \boldsymbol{B})=0$ in the general case does not imply the equality of multisets $\boldsymbol{A}$ and $\boldsymbol{B}$. Thus, only the coincidence condition $\left(4^{0}\right)$ holds for function $d_{Z_{4 p}}(\boldsymbol{A}, \boldsymbol{B})$. Additionally, for multisets that differ by a multiset of measure zero, condition $m(\boldsymbol{A} \Delta \boldsymbol{B})=0$ implies the $m$-equality of multisets $\boldsymbol{A}$ and $\boldsymbol{B}$, and hence the identity axiom $\left(2^{0}\right)$ also holds. Thus, function $d_{Z_{4 p}}(\boldsymbol{A}, \boldsymbol{B})$ is quasimetric and almost everywhere sym- 
metric on $\sigma$-algebra $S_{\sigma}(Z)$ of measurable multisets with a strongly $\sigma$-additive and completely $\sigma$-finite measure.

\section{ACKNOWLEDGMENTS}

This work was supported by the Russian Foundation for Basic Research (projects nos. 15-07-04760, 16-07-01125, 16-29-12864, and 17-07-00512) and the Russian Academy of Sciences Presidium (the program of fundamental research "Intelligent Information Technologies and Systems").

\section{REFERENCES}

1. Lyusternik, L.A. and Sobolev, V.I., Elementy funktsional'nogo analiza (Elements of Functional Analysis), Moscow: Nauka, 1965.

2. Kolmogorov, A.N. and Fomin, S.V., Elementy teorii funktsiy i funktsional'nogo analiza (Elements of the Theory of Functions and Functional Analysis), Moscow: Nauka, 1968.

3. Deza, M.M. and Laurent, M., Geometry of Cuts and Metrics, Berlin: Springer-Verlag, 1997.

4. Sneath, P.H.A. and Sokal, R.R., Numerical taxonomy, in The Principles and Practice of Numerical Classification, San Francisco: Freeman, 1973.

5. Hartigan, J.A., Clustering Algoritms, New York: Wiley, 1975.

6. Petrovsky, A.B., Prostranstva mnozhestv $i$ mul'timnozhestv (Spaces of Sets and Multisets), Moscow: Editorial URSS, 2003.

7. Petrovsky, A.B., Prostranstva izmerimykh mnozhestv $i$ mul'timnozhestv (Spaces of Measurable Sets and Multisets), Moscow: Poli Print Servis, 2016.

8. Petrovsky, A.B., On distances between sets invariant under space transformations, I Vsesoyuznoye soveshchaniye po statisticheskomu i diskretnomu analizu nechislovoi informatsii, ekspertnym otsenkam i diskretnoy optimizatsii. Tezisy dokladov (I All-Union Meeting on Statistical and Discrete Analysis of Non-Numerical Information, Expert Assessments, and Discrete Optimization. Proceedings), Moscow-Alma-Ata, 1981, pp. 50-51.
9. Petrovsky, A.B., An axiomatic approach to metrization of multiset space, in Multiple Criteria Decision Making, Tzeng, G.H., Wang, H.F., Wen, U.P., and Yu, P.L., Eds., New York: Springer-Verlag, 1994, pp. 129-140.

10. Petrovsky, A.B., Metric spaces of multisets, Dokl. Akad. Nauk, 1995, vol. 344, no. 2, pp. 175-177.

11. Petrovsky, A.B., Combinatorics of multisets, Dokl. Akad. Nauk, 2000, vol. 370, no. 6, pp. 750-753.

12. Petrovsky, A.B., Multisets as a model for representation of multi-attribute objects in decision-making and pattern recognition, Iskusstv. Intell., 2002, no. 2, pp. 236243.

13. Petrovsky, A., Group verbal decision analysis, in Encyclopedia of Decision Making and Decision Support Technologies, Adam, F. and Humphreys, P., Eds., Hershey, New York: IGI Global, 2008, vol. 1, pp. 418-425.

14. Petrovsky, A., Multi-attribute classification of credit cardholders: Multiset approach, Int. J. Manage. Decis. Making, 2006, vol. 7, nos. 2-3, pp. 66-179.

15. Petrovsky, A.B., Methods for the group classification of multi-attribute objects (part 1, part 2), Sci. Tech. Inf. Process., 2010, vol. 37, no. 5, pp. 346-356, 357-368.

16. Petrovsky, A., Group multiple criteria decision making: Multiset approach, in Recent Developments and New Directions in Soft Computing. Studies in Fuzziness and Soft-Computing, Springer International Publishing, 2014, pp. 19-33.

17. Petrovsky, A.B. and Lobanov, V.N., Multi-criteria choice in the attribute space of large dimension: Multimethod technology PAKS-M, Sci. Tech. Inf. Process., 2014, no. 3, pp. 92-104.

18. Syomkin, B.I. and Dvoychenkov, V.I., On the equivalence of measures of similarity and difference, in Issledovaniye sistem (Research of Systems), vol. 1: Analiz slozhnykh sistem (Analysis of Complex Systems), Vladivostok: Dal'nevost. Nauchn. Tsentr Akad. Nauk SSSR, 1973, pp. 95-104.

19. Kosters, W.A. and Laros, J.F.J., Metrics for multisets, Research and Development in Intelligent Systems XXIV, Proceedings of AI-2007, Springer-Verlag London Limited, 2008, pp. 294-303.

Translated by K. Lazarev 